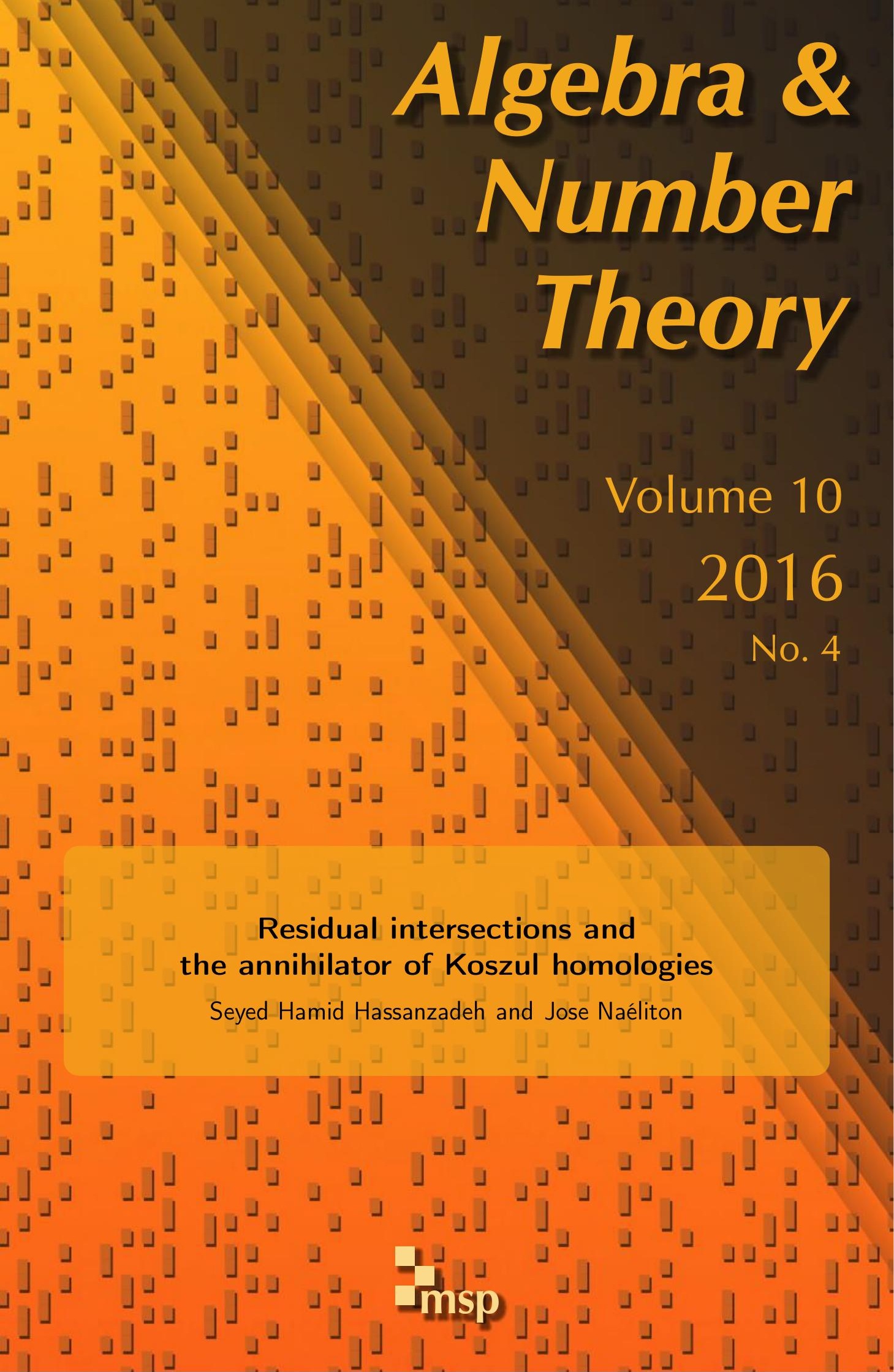




\title{
Residual intersections and the annihilator of Koszul homologies
}

\author{
Seyed Hamid Hassanzadeh and Jose Naéliton
}

\begin{abstract}
We study Cohen-Macaulayness, unmixedness, the structure of the canonical module and the stability of the Hilbert function of algebraic residual intersections. We establish some conjectures about these properties for large classes of residual intersections without restricting the local number of generators of the ideals involved. To determine the above properties, we construct a family of approximation complexes for residual intersections. Moreover, we determine some general properties of the symmetric powers of quotient ideals which were not known even for special ideals with a small number of generators. Finally, we show acyclicity of a prime case of these complexes to be equivalent to finding a common annihilator for higher Koszul homologies, which unveils a tight relation between residual intersections and the uniform annihilator of positive Koszul homologies, shedding some light on their structure.
\end{abstract}

\section{Introduction}

Understanding the residual of the intersection of two algebraic varieties is an intriguing concept in algebraic geometry. Similar to many other notions in intersection theory, the concept of residual intersections needs the proper interpretation to encompass the desired algebraic and geometric properties. In the sense of [Artin and Nagata 1972], which is our point of view, the notion is a vast generalization of linkage (or liaison), which is more ubiquitous, but also harder to understand. Let $X$ and $Y$ be irreducible closed subschemes of a Noetherian scheme $Z$ with $\operatorname{codim}_{Z}(X) \leq \operatorname{codim}_{Z}(Y)=s$ and $Y \not \subset X$. Then $Y$ is called a residual intersection of $X$ if the number of equations needed to define $X \cup Y$ as a subscheme of $Z$ is the smallest possible, that is, $s$. Precisely, if $R$ is a Noetherian ring, $I$ an ideal of height $g$ and $s \geq g$ an integer, then:

Hassanzadeh was partially supported by a CNPq grant (300586/2012-4) and Naéliton worked under a CNPq Doctoral scholarship.

MSC2010: primary 13C40; secondary 13H10, 13D02, 14C17, 14M06.

Keywords: residual intersection, canonical module, type, sliding depth, approximation complex,

Koszul annihilator. 
- An (algebraic) $s$-residual intersection of $I$ is a proper ideal $J$ of $R$ such that $\operatorname{ht}(J) \geq s$ and $J=\left(\mathfrak{a}:_{R} I\right)$ for some ideal $\mathfrak{a} \subset I$ generated by $s$ elements.

- A geometric s-residual intersection of $I$ is an algebraic $s$-residual intersection $J$ of $I$ such that ht $(I+J) \geq s+1$.

Based on a construction of Laksov for residual intersection, Fulton [1998, Definition 9.2.2] presents a formulation for residual intersection that, locally, can be expressed as follows: suppose that $X=\operatorname{Spec}(R)$ and that $Y$ and $S$ are closed subschemes of $X$ defined by the ideals $\mathfrak{a}$ and $I$, respectively. Let $\tilde{X}=\operatorname{Proj}\left(\mathcal{R}_{R}(I)\right)$ be the blow-up of $X$ along $S$. Consider the natural map $\pi: \tilde{X} \rightarrow X$. Let $\tilde{Y}=\pi^{-1}(Y)$ and $\tilde{S}=\pi^{-1}(S)$. Then $\tilde{Y}=\operatorname{Proj}\left(\mathcal{R}_{R}(I) / \mathfrak{a} \mathcal{R}_{R}(I)\right)$ and $\tilde{S}=\operatorname{Proj}\left(\mathcal{R}_{R}(I) / I \mathcal{R}_{R}(I)\right)$ are closed subschemes of $\tilde{X}$ with ideal sheaves $\mathcal{I}_{\tilde{Y}}$ and $\mathcal{I}_{\tilde{S}}$, where $\mathcal{I}_{\tilde{S}}$ is an invertible sheaf. Let $Z^{\prime} \subseteq \tilde{X}$ be the closed subscheme defined by the ideal sheaf $\mathcal{I}_{Z^{\prime}}=\mathcal{I}_{\tilde{Y}} \cdot \mathcal{I}_{\tilde{S}}^{-1}$. Then $Z^{\prime}$ is called the residual scheme to $\tilde{S}$ in $\tilde{Y}$. Precisely $Z^{\prime}=\operatorname{Proj}\left(\mathcal{R}_{R}(I) / \gamma \mathcal{R}_{R}(I)\right)$ in which $\gamma=\mathfrak{a} \subseteq\left(\mathcal{R}_{R}(I)\right)_{[1]}$. Finally, the residual intersection to $S$ in $Y$ [Fulton 1998, Definition 9.2.2] is the direct image of $\mathcal{O}_{Z^{\prime}}$, i.e., $\pi_{*}\left(\mathcal{O}_{Z^{\prime}}\right)$. Since $\mathcal{O}_{Z^{\prime}}$ is a coherent sheaf, by [Hartshorne 1977 , Chapter III, Proposition 8.5],

$$
\pi_{*}\left(\mathcal{O}_{Z^{\prime}}\right)=\left(H^{0}\left(\tilde{X}, \mathcal{O}_{Z^{\prime}}\right)\right)^{\tilde{z}}=\left(\Gamma\left(\tilde{X}, \mathcal{O}_{Z^{\prime}}\right)\right)^{\tilde{2}} .
$$

Note that $\Gamma\left(\tilde{X}, \mathcal{O}_{Z^{\prime}}\right)$ equals $\Gamma_{*}\left(\tilde{X}, \mathcal{O}_{Z^{\prime}}(0)\right)$, which is equal to the ideal transform $D_{\mathcal{R}_{R}(I)_{+}}\left(\mathcal{R}_{R}(I) / \gamma \mathcal{R}_{R}(I)\right)_{[0]}$, by an application of the Čech complex. The latter is closely related, and in many cases determined, by the ideal

$$
H_{\mathcal{R}_{R}(I)_{+}}^{0}\left(\mathcal{R}_{R}(I) / \gamma \mathcal{R}_{R}(I)\right)_{[0]}=\bigcup\left(\mathfrak{a} I^{i}:_{R} I^{i+1}\right) .
$$

In [Hassanzadeh 2012] and in the current work, we consider the symmetric algebra $\operatorname{Sym}_{R}(I)$ instead of the Rees algebra $\mathcal{R}_{R}(I)$. This generalization has already proved its usefulness in studying multiple-point formulas by Kleiman; see [Fulton 1998, Example 17.6.2]. We then find a kind of arithmetical residual intersection to be $\bigcup\left(\gamma \operatorname{Sym}_{R}^{i}(I):_{R} \operatorname{Sym}_{R}^{i+1}(I)\right)$, where $\gamma=\mathfrak{a} \subseteq \operatorname{Sym}_{R}^{i}(I)_{[1]}$. Comparing the above three definitions for residual intersection, we have

$$
J=(\mathfrak{a}: I) \subseteq \bigcup\left(\gamma \operatorname{Sym}_{R}^{i}(I):_{R} \operatorname{Sym}_{R}^{i+1}(I)\right) \subseteq \bigcup\left(\mathfrak{a} I^{i}:_{R} I^{i+1}\right) .
$$

Interestingly, these ideals coincide if the algebraic residual intersection $J$ does not share any associated primes with $I$, e.g., if $J$ is unmixed and the residual is geometric.

Determining the cases where the first inclusion above is an equality leads us to define a third variation of algebraic residual intersection.

Definition 1.1. An arithmetic $s$-residual intersection $J=(\mathfrak{a}: I)$ is an algebraic $s$-residual intersection such that $\mu_{R_{\mathfrak{p}}}\left((I / \mathfrak{a})_{\mathfrak{p}}\right) \leq 1$ for all prime ideals $\mathfrak{p} \supseteq(I+J)$ with $h t(\mathfrak{p}) \leq s$. (Here $\mu$ denotes the minimum number of generators.) 
Clearly any geometric $s$-residual intersection is arithmetic. Moreover, for any algebraic $s$-residual intersection $J=\left(\mathfrak{a}:\left(f_{1}, \ldots, f_{r}\right)\right)$ which is not geometric, all of the colon ideals $\left(\mathfrak{a}: f_{i}\right)$ are arithmetic $s$-residual intersections and at least one of them is not geometric.

In this paper we introduce a family of complexes, denoted by $\left\{\mathcal{Z}_{\cdot}^{+}\right\}_{i=0}^{\infty}$, to approximate the $i$-th symmetric power of $I / \mathfrak{a}$, which is denoted by $\operatorname{Sym}_{R}^{i}(I / \mathfrak{a})$ for $i>1$. The idea to define this family is inspired by [Hassanzadeh 2012] in which the single complex ${ }_{0} \mathcal{Z}^{+}$is treated. $H_{0}\left({ }_{0} \mathcal{Z}_{\bullet}^{+}\right)$is a cyclic module of the form $R / K$, where $K$ is called the disguised s-residual intersection of $I$ with respect to $\mathfrak{a}$; see Definition 2.1. The study of the other members of the above family of complexes sheds some more light on the structure of residual intersections. A flavor of our main results in Section 2 is the following:

Main results. Let $(R, \mathfrak{m})$ be a Cohen-Macaulay $(C M)$ local ring of dimension d and let $I$ be an ideal with $\mathrm{ht}(I)=g>0$. Let $s \geq g$ and $1 \leq k \leq s-g+2$ and let $J=(\mathfrak{a}: I)$ be any (algebraic) $s$-residual intersection. Suppose that I is strongly Cohen-Macaulay (SCM). Then:

(1) (Theorem 2.14) The canonical module of $R / J$ is $\operatorname{Sym}_{R}^{s-g+1}(I / \mathfrak{a})$, provided the residual is arithmetic and $R$ is Gorenstein.

(2) $($ Corollary 2.8) $\operatorname{depth}(R / \mathfrak{a})=d-s$.

(3) (Corollary 2.8) $J$ is unmixed of codimension $s$.

(4) (Theorem 2.6 and Proposition 3.1) ${ }_{k} \mathcal{Z}_{\text {. }}^{+}$is acyclic, $H_{0}\left({ }_{k} \mathcal{Z}_{\bullet}^{+}\right)=\operatorname{Sym}_{R}^{k}(I / \mathfrak{a})$ and the latter is $C M$ of dimension $d-s$ (the acyclicity of $\mathcal{Z}_{\text {. }}^{+}$implies conjecture (5) below in the arithmetic case).

These results address the following (implicit) conjectures made during the development of the theory of algebraic residual intersections.

Conjectures. Let $R$ be a CM local ring and let I be SCM, or even just satisfying sliding depth $(S D)$. Then:

(1) $R / J$ is Cohen-Macaulay.

(2) The canonical module of $R / J$ is the $(s-g+1)$-st symmetric power of $I / \mathfrak{a}$, if $R$ is Gorenstein.

(3) $\mathfrak{a}$ is minimally generated by s elements.

(4) $J$ is unmixed.

(5) The Hilbert series of $R / J$ depends only on I and the degrees of the generators of $\mathfrak{a}$.

The first conjecture essentially goes back to [Artin and Nagata 1972], and it has been asked as an open question in [Huneke and Ulrich 1988]. At the Sundance 
conference in 1990, Ulrich [1992] mentioned conjectures (1)-(4) above as desirable facts to be proved. The property of the Hilbert function is rather recent and has been analyzed by Chardin, Eisenbud and Ulrich [Chardin et al. 2001; Chardin et al. 2015].

It should be mentioned that these conjectures are proved if one supposes in addition that the ideal $I$ has locally few generators, a condition which is called $G_{s}$, or if it has a deformation with the $G_{s}$ property. Over time the $G_{s}$ condition has become a "standard" assumption in the theory of residual intersections which is not avoidable in some cases. However, the desire is to prove the above assertions without restricting the local number of generators of $I$.

In comparison, obtaining the structure of the canonical module in the absence of the $G_{s}$ condition is more challenging. To achieve this, we show that under the above hypotheses, $\operatorname{Sym}_{R}^{s-g+1}(I / \mathfrak{a})$ is a faithful maximal Cohen-Macaulay $R / J$-module of type 1 . Concerning the type of modules, we prove even more. We show in Theorem 2.12 that the inequality

$$
r_{R}\left(\operatorname{Sym}_{R}^{k}(I / \mathfrak{a})\right) \leq\left(\begin{array}{c}
r+s-g-k \\
r-1
\end{array}\right) r_{R}(R)
$$

holds for any $1 \leq k \leq s-g+1$, where, for a finitely generated $R$-module $M$, $r_{R}(M):=\operatorname{dim}_{R / \mathfrak{m}} \operatorname{Ext}_{R}^{\operatorname{depth}(M)}(R / \mathfrak{m}, M)$ is the Cohen-Macaulay type of $M$.

In Section 3, we present several applications of the theorems and constructions so far. We state how much the Hilbert functions of $R / J$ and $R / \mathfrak{a}$ depend on the generators and/or degrees of $I$ and $\mathfrak{a}$. If $I$ is SCM, then the Hilbert function of the disguised residual intersection, and that $\operatorname{Sfym}_{R}^{k}(I / \mathfrak{a})$, if $1 \leq k \leq s-g+2$, depends only on the degrees of the generators of $\mathfrak{a}$ and the Koszul homologies of $I$. In particular, $k=1$ implies that the Hilbert function of $R / \mathfrak{a}$ is constant on the open set of ideals $\mathfrak{a}$ generated by $s$ forms of the given degrees such that $h t(\mathfrak{a}: I) \geq s$. This is comparable with results in [Chardin et al. 2001], where the same assertion is concluded under some $G_{s}$ hypotheses.

The graded structure of ${ }_{k} \mathcal{Z}^{+}$shows that if $I$ satisfies the $\mathrm{SD}_{1}$ condition, then

$$
\operatorname{reg}\left(\operatorname{Sym}_{R}^{k}(I / \mathfrak{a})\right) \leq \operatorname{reg}(R)+\operatorname{dim}\left(R_{0}\right)+\sigma(\mathfrak{a})-(s-g+1-k) \operatorname{indeg}(I / \mathfrak{a})-s
$$

for $k \geq 1$. These applications were not known even when ideal $I$ satisfies the $G_{s}$ condition. Finally, in Proposition 3.4, by a combination of older and newer facts, we show that for any algebraic $s$-residual intersection $J=\mathfrak{a}: I$, if $I$ is SCM and evenly linked to a $G_{s}$ ideal (or has a deformation with these properties) then the disguised residual intersection and the algebraic residual intersection coincide. Based on this fact, we conjecture that (Conjecture 5.9) in the presence of the sliding depth condition, the disguised residual intersection is the same as the algebraic residual intersection. 
In Section 4, we try to understand better the structure of the complex ${ }_{0} \mathcal{Z}^{+}$in the case where $I / \mathfrak{a}$ is principal, e.g., $I=(\mathfrak{a}, b)$. We find in Theorem 4.4 that $H_{i}\left({ }_{0} \mathcal{Z}_{\bullet}^{+}(\boldsymbol{a}, \boldsymbol{f})\right) \simeq b H_{i}\left(a_{1}, \ldots, a_{s}\right)$ for all $i \geq 1$ and $H_{0}\left({ }_{0} \mathcal{Z}_{\bullet}^{+}(\boldsymbol{a}, \boldsymbol{f})\right) \simeq R /(\mathfrak{a}: b)$. This fact shows how much the homologies of $\mathcal{Z}_{\text {. }}^{+}$complexes may depend on the generating sets, as well as showing a tight relation between the uniform annihilator of Koszul homologies and acyclicity of $\mathcal{Z}_{\text {. }}^{+}$. As a byproduct, Corollary 4.6 suggests that to study the properties of colon ideals, instead of assuming ht $\left(\left(a_{1}, \ldots, a_{s}\right): I\right) \geq s$, one may only need to suppose that $I H_{i}\left(a_{1}, \ldots, a_{s}\right)=0$ locally at codimension $s-1$.

Motivated by the facts in Section 4, we investigate the uniform annihilator of positive Koszul homologies in Section 5. Not much is known about the annihilator of Koszul homologies. In Corollary 5.6, we show that for a residual intersection $J=\mathfrak{a}: I$, where $I$ satisfies SD and $\operatorname{depth}(R / I) \geq d-s$,

$$
I \subseteq \bigcap_{j \geq 1} \operatorname{Ann}\left(H_{j}(\mathfrak{a})\right) .
$$

Surprisingly, this result contradicts one of the unpublished but well-known results of G. Levin [Vasconcelos 2005, Theorem 5.26], which yielded in [Corso et al. 2006] that $\operatorname{Supp}\left(H_{1}(\mathfrak{a})\right)=\operatorname{Supp}\left(H_{0}(\mathfrak{a})\right)$. Simple examples of residual intersection disprove this last claim. Moreover, we show in Theorem 5.4 that for an $s$-residual intersection $J=(\mathfrak{a}: I)$, if $I$ satisfies $\operatorname{SD}$ and $\operatorname{depth}(R / I) \geq d-s$, so does $\mathfrak{a}$. This is an interesting result since for a long time it was known that the residual intersections of the ideal $\mathfrak{a}$ are Cohen-Macaulay although no one was aware of the SD property of $\mathfrak{a}$.

\section{Residual approximation complexes}

In this section we introduce a family of complexes which approximate the residual intersection and some of its related symmetric powers. We denote this family by $\left\{{ }_{i} \mathcal{Z}_{\bullet}^{+}\right\}_{i=0}^{\infty}$. The complex ${ }_{0} \mathcal{Z}_{\text {. }}^{+}$was already defined in [Hassanzadeh 2012] and used to prove the CM-ness of arithmetic residual intersections of ideals with sliding depth.

Throughout this section, $R$ is a Noetherian ring of dimension $d$, and $I=(f)=$ $\left(f_{1}, \ldots, f_{r}\right)$ is an ideal of grade $g \geq 1$. Although by adding one variable we could also treat the case $g=0$, for simplicity we keep the assumption $g \geq 1$. Let $\mathfrak{a}=\left(a_{1}, \ldots, a_{s}\right)$ be an ideal contained in $I$, with $s \geq g$, let $J=\mathfrak{a}:_{R} I$, and let $S=R\left[T_{1}, \ldots, T_{r}\right]$ be a polynomial extension of $R$ with indeterminates $T_{i}$. We denote the symmetric algebra of $I$ over $R$ by $\mathcal{S}_{I}$ or, in general, the symmetric algebra of an $R$-module $M$ by $\operatorname{Sym}_{R}(M)$ and the $k$-th symmetric power of $M$ by $\operatorname{Sym}_{R}^{k}(M)$. We consider $\mathcal{S}_{I}$ as an $S$-algebra via the ring homomorphism $S \rightarrow \mathcal{S}_{I}$ sending $T_{i}$ to $f_{i}$ as an element of $\left(\mathcal{S}_{I}\right)_{1}=I$; then $\mathcal{S}_{I}=S / \mathcal{L}$. Let $a_{i}=\sum_{j=1}^{r} c_{j i} f_{j}$, $\gamma_{i}=\sum_{j=1}^{r} c_{j i} T_{j}, \gamma=\left(\gamma_{1}, \ldots, \gamma_{s}\right)$ and $\mathfrak{g}:=\left(T_{1}, \ldots, T_{r}\right)$.

For a sequence of elements $\mathfrak{x}$ in a commutative ring $A$ and an $A$-module $M$, we denote the Koszul complex by $K_{\bullet}(\mathfrak{x} ; M)$, its cycles by $Z_{i}(\mathfrak{x} ; M)$ and its homologies 
by $H_{i}(\mathfrak{x} ; M)$. For a graded module $M, \operatorname{indeg}(M):=\inf \left\{i: M_{i} \neq 0\right\}$ and end $(M):=$ $\sup \left\{i: M_{i} \neq 0\right\}$. Setting $\operatorname{deg}\left(T_{i}\right)=1$ for all $i, S$ is a standard graded ring over $S_{0}=R$.

To set one more convention, when we draw the picture of a double complex obtained from a tensor product of two finite complexes (in the sense of [Weibel 1994, 2.7.1]), say $\mathcal{A} \otimes \mathcal{B}$, we always put $\mathcal{A}$ in the vertical direction and $\mathcal{B}$ in the horizontal one. We also label the module which is in the upper-right corner as $(0,0)$ and consider the labels for the rest as the points in the third quadrant.

${ }_{k} \mathcal{Z}_{\bullet}^{+}$complexes. The first object in the construction of the family of ${ }_{k} \mathcal{Z}^{+}$complexes is one of the approximation complexes - the $\mathcal{Z}$-complex [Herzog et al. 1983]. We consider the approximation complex $\mathcal{Z}_{\bullet}(\boldsymbol{f})$,

$$
0 \rightarrow Z_{r-1} \otimes_{R} S(1-r) \rightarrow \cdots \rightarrow Z_{1} \otimes_{R} S(-1) \rightarrow Z_{0} \otimes_{R} S \rightarrow 0,
$$

where $Z_{i}=Z_{i}(f)$ is the $i$-th cycle of the Koszul complex $K \cdot(f, R)$.

The second object is the Koszul complex $K_{\bullet}(\gamma, S)$,

$$
0 \rightarrow K_{s}\left(\gamma_{1}, \ldots, \gamma_{s}\right)(-s) \rightarrow \cdots \rightarrow K_{1}\left(\gamma_{1}, \ldots, \gamma_{s}\right)(-1) \rightarrow K_{0}\left(\gamma_{1}, \ldots, \gamma_{s}\right) \rightarrow 0
$$

Let $\mathcal{D}_{\bullet}=\operatorname{Tot}\left(K_{\bullet}(\boldsymbol{\gamma}, S) \otimes_{S} \mathcal{Z}_{\bullet}(\boldsymbol{f})\right)$. Then

$$
\mathcal{D}_{i}=\bigoplus_{j=i-s}^{\min \{i, r-1\}}\left[Z_{j} \otimes_{R} S\right]^{\left(\begin{array}{c}
{ }^{s}-j \\
)
\end{array}\right)}(-i) .
$$

For a graded $S$-module $M$, the $k$-th graded component of $M$ is denoted by $M_{[k]}$. Let $\left(D_{\bullet}\right)_{[k]}$ for $k \in \mathbb{Z}$ be the $k$-th graded strand of $D_{\text {. }}$. We have $\left(D_{i}\right)_{[k]}=0$ for all $k<i$; in particular,

$$
H_{k}\left(\left(D_{\bullet}\right)_{[k]}\right)=\operatorname{Ker}\left(D_{k} \rightarrow D_{k-1}\right)_{[k]} .
$$

Now, let $C_{\mathfrak{g}}^{\bullet}=C_{\mathfrak{g}}^{\bullet}(S)$ be the Čech complex of $S$ with respect to the sequence $\mathfrak{g}=\left(T_{1}, \ldots, T_{r}\right)$,

$$
C_{\mathfrak{g}}^{\bullet}: 0 \rightarrow C_{\mathfrak{g}}^{0}(=S) \rightarrow C_{\mathfrak{g}}^{1} \rightarrow \cdots \rightarrow C_{\mathfrak{g}}^{r} \rightarrow 0 .
$$

We then consider the bicomplex $C_{\mathfrak{g}}^{\bullet} \otimes_{S} D$. with $C_{\mathfrak{g}}^{0} \otimes_{S} D_{0}$ in the corner. This bicomplex gives rise to two spectral sequences for which the second terms of the horizontal spectral are

$$
{ }^{2} \mathrm{E}_{\mathrm{hor}}^{-i,-j}=H_{\mathfrak{g}}^{j}\left(H_{i}\left(D_{\bullet}\right)\right),
$$

and the first terms of the vertical spectral are

$$
\mathrm{E}_{\mathrm{ver}}^{-i,-j}= \begin{cases}0 \rightarrow H_{\mathfrak{g}}^{r}\left(D_{r+s-1}\right) \rightarrow \cdots \rightarrow H_{\mathfrak{g}}^{r}\left(D_{1}\right) \rightarrow H_{\mathfrak{g}}^{r}\left(D_{0}\right) \rightarrow 0 & \text { if } j=r, \\ 0 & \text { otherwise }\end{cases}
$$


Since we have $H_{\mathfrak{g}}^{r}\left(D_{i}\right)=H_{\mathfrak{g}}^{r}\left(\bigoplus_{j}\left[Z_{j} \otimes S\right](-i)\right)=\bigoplus_{j} Z_{j} \otimes H_{\mathfrak{g}}^{r}(S)(-i)$ and end $\left(H_{\mathfrak{g}}^{r}(S)\right)=-r$, it follows that end $\left(H_{\mathfrak{g}}^{r}\left(D_{i}\right)\right)=i-r$, thus $H_{\mathfrak{g}}^{r}\left(D_{i}\right)_{[i-r+j]}=0$, for all $j \geq 1$. We then define the following sequence of complexes indexed by $k \geq 0$ :

$$
0 \rightarrow H_{\mathfrak{g}}^{r}\left(D_{r+s-1}\right)_{[k]} \rightarrow \cdots \rightarrow H_{\mathfrak{g}}^{r}\left(D_{r+k+1}\right)_{[k]} \stackrel{\phi_{k}}{\longrightarrow} H_{\mathfrak{g}}^{r}\left(D_{r+k}\right)_{[k]} \rightarrow 0 .
$$

Since the vertical spectral (2-4) collapses at the second step, the horizontal spectral converges to the homologies of $H_{\mathfrak{g}}^{r}\left(D_{\bullet}\right)$. Since all of the homomorphisms are homogeneous of degree 0 , the convergence inherits to any graded component. Therefore, for any $k \geq 0$, there exists a filtration $\cdots \subseteq \mathcal{F}_{2 k} \subseteq \mathcal{F}_{1 k} \subseteq \operatorname{Coker}\left(\phi_{k}\right)$ such that

$$
\frac{\operatorname{Coker}\left(\phi_{k}\right)}{\mathcal{F}_{1 k}} \simeq\left({ }^{\infty} \mathrm{E}_{\mathrm{hor}}^{-k, 0}\right)_{[k]} .
$$

Observing that $H_{\mathfrak{g}}^{-t}\left(H_{h}\left(D_{\bullet}\right)\right)=0$ for all $(t, h) \in \mathbb{N} \times \mathbb{N}_{0}$, one has ${ }^{l} \mathrm{E}_{\text {hor }}^{-k, 0} \subseteq$ $H_{\mathfrak{g}}^{0}\left(H_{k}\left(D_{\bullet}\right)\right)$ for all $l \geq 2$. Hence we have the following chain of maps for which, except the isomorphism in the middle, all maps are canonical and the map on the right is given by (2-2):

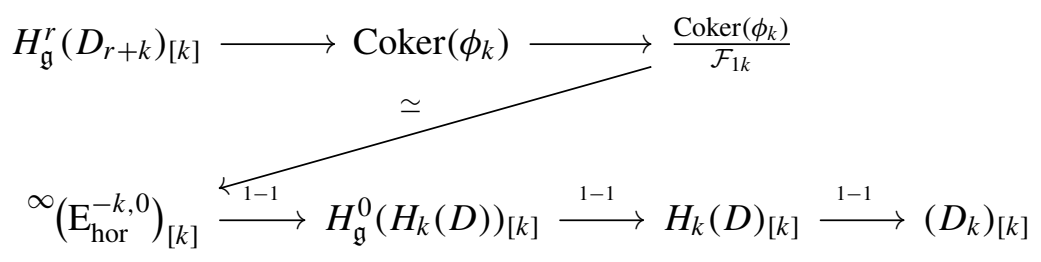

We denote the composition of the above chain of $R$-homomorphisms by $\tau_{k}$.

Finally, we define the promised family of complexes as follows. For any integer $k \geq 0,{ }_{k} \mathcal{Z}_{\text {. }}^{+}$is a complex of length $s$ consisting of two parts: the right part is $\left(D_{\bullet}\right)_{[k]}$ and the left part is $\left({ }^{1} \mathrm{E}_{\mathrm{ver}}\right)_{[k]}$. These parts are joined via $\tau_{k}$. More precisely,

$$
{ }_{k} \mathcal{Z}_{\bullet}^{+}: 0 \rightarrow{ }_{k} \mathcal{Z}_{s}^{+} \rightarrow \cdots \stackrel{\phi_{k}}{\longrightarrow}{ }_{k} \mathcal{Z}_{k+1}^{+} \stackrel{\tau_{k}}{\longrightarrow}{ }_{k} \mathcal{Z}_{k}^{+} \rightarrow \cdots \rightarrow{ }_{k} \mathcal{Z}_{0}^{+} \rightarrow 0,
$$

wherein

$$
{ }_{k} \mathcal{Z}_{i}^{+}= \begin{cases}\left(D_{i}\right)_{[k]}, & i \leq \min \{k, s\}, \\ H_{\mathfrak{g}}^{r}\left(D_{i+r-1}\right)_{[k]}, & i>k .\end{cases}
$$

The structure of ${ }_{k} \mathcal{Z}_{\text {. }}^{+}$depends in two ways on the generating sets. Namely, it depends on the generating set of $I$, which is $f$, and the expression of the generators of $\mathfrak{a}$ in terms of the generators of $I$, which are given by $c_{i j}$. However, for $k \geq 1$, we have

$$
H_{0}\left({ }_{k} \mathcal{Z}_{\bullet}^{+}\right)=H_{0}\left(D_{\bullet}\right)_{[k]}=\left(\mathcal{S}_{I} /(\gamma) \mathcal{S}_{I}\right)_{[k]}=\operatorname{Sym}_{R}^{k}(I / \mathfrak{a}) .
$$

The case where $k=0$ is also very interesting. However, the structure of $H_{0}\left({ }_{0} \mathcal{Z}_{\bullet}^{+}\right)$ is not as clear as the cases where $k>0$. 
Definition 2.1. Let $R$ be a Noetherian ring and let $\mathfrak{a} \subseteq I$ be two ideals of $R$. The disguised s-residual intersection of I with respect to $\mathfrak{a}$ is the unique ideal $K$ such that $H_{0}\left({ }_{0} \mathcal{Z}^{+}\right)=R / K$. The reasons for choosing the attribute disguised are as follows: $K$ is contained in $J=(\mathfrak{a}: I)$ and it has the same radical as $J$ has; if $R$ is CM, $J$ is an algebraic residual intersection and $I$ satisfies some sliding depth condition, then $K$ is Cohen-Macaulay; moreover, $K$ coincides with $J$ in the case where the residual is arithmetic, by [Hassanzadeh 2012, Theorem 2.11]. We conjecture (Conjecture 5.9) that under the above assumptions $K$ is always the same as $J$ despite that it does not appear so.

Acyclicity and Cohen-Macaulayness. One more occasion where the properties of ${ }_{k} \mathcal{Z}^{+}$are independent of the generating sets of $I$ and $\mathfrak{a}$ is the following lemma, which is crucial to proving the acyclicity of ${ }_{k} \mathcal{Z}_{\bullet}^{+}$.

Lemma 2.2. Let $R$ be a Noetherian ring and let $\mathfrak{a} \subseteq I$ be two ideals of $R$. If $I=\mathfrak{a}$,

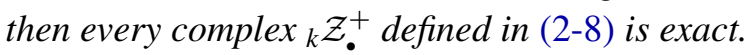

Proof. Since the approximation complex $\mathcal{Z}_{\bullet}(\boldsymbol{f})$ is a differential graded algebra, $H_{i}\left(\mathcal{Z}_{\text {. }}\right)$ is an $\mathcal{S}_{I}=S / \mathcal{L}$-module for all $i$. Likewise, since the approximation complex $K_{\bullet}(\boldsymbol{\gamma})$ is a differential graded algebra, $H_{i}\left(K_{\bullet}(\boldsymbol{\gamma})\right)$ is an $S /(\boldsymbol{\gamma})$-module for all $i$. The bicomplex $K_{\bullet}(\boldsymbol{\gamma}) \otimes_{S} \mathcal{Z}_{\text {. gives rise to the horizontal spectral sequence with second }}$ terms ${ }^{2} \mathrm{E}_{\text {hor }}^{-i,-j}=H_{j}\left(K_{\bullet}\left(\boldsymbol{\gamma} ; H_{i}\left(\mathcal{Z}_{\bullet}\right)\right)\right)$. It follows that $(\mathcal{L}+(\boldsymbol{\gamma}))$ annihilates ${ }^{2} \mathrm{E}_{\text {hor }}^{-i,-j}$ and consequently annihilates ${ }^{\infty} \mathrm{E}_{\mathrm{hor}}^{-i,-j}$, which is a subquotient of ${ }^{2} \mathrm{E}_{\mathrm{hor}}^{-i,-j}$. By the convergence of the spectral sequence to the homologies of the total complex $H_{\bullet}\left(D_{\text {。 }}\right)$, it is straightforward to deduce that $H_{i}\left(D_{\boldsymbol{\bullet}}\right)$ is an $(\mathcal{L}+(\boldsymbol{\gamma}))$-torsion module for all $i$, i.e., $(\mathcal{L}+(\boldsymbol{\gamma}))^{N} H_{i+j}\left(D_{\bullet}\right)=0$ for all $i, j$ and for some $N$. Considering the equation $I=\mathfrak{a}$ in $\left(\mathcal{S}_{I}\right)_{1}$, we have $\mathcal{L}+(\boldsymbol{\gamma})=\mathcal{L}+\mathfrak{g}$. Therefore,

$$
H_{\mathfrak{g}}^{j}\left(H_{i}\left(D_{\bullet}\right)\right)=H_{\mathfrak{g}+\mathcal{L}}^{j}\left(H_{i}\left(D_{\bullet}\right)\right)=H_{(\gamma)+\mathcal{L}}^{j}\left(H_{i}\left(D_{\bullet}\right)\right)= \begin{cases}H_{i}\left(D_{\bullet}\right), & j=0, \\ 0, & j>0 .\end{cases}
$$

Once more we study the spectral sequences arising from $C_{\mathfrak{g}} \otimes D_{\bullet}$, which were already mentioned in (2-8). Applying (2-10), we draw both horizontal and vertical spectral sequences simultaneously as follows:

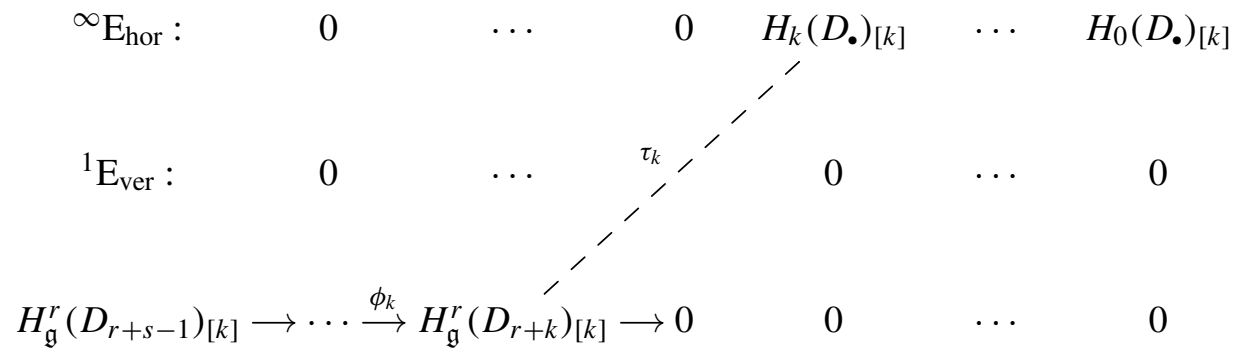


Since $H_{j}\left(D_{\bullet}\right)$ is a subquotient of $D_{j}$, we have $H_{j}\left(D_{\bullet}\right)_{[k]}=0$ for all $j>k$. Also, by $(2-10)$ there is only one nonzero row in the horizontal spectral. On the other hand, $\mathfrak{g}$ is a regular sequence on the $D_{j}$ which in turn shows that the vertical spectral is just one line.

Consequently, $H_{j}\left({ }_{k} \mathcal{Z}_{\bullet}^{+}\right):=H_{j+r-1}\left(H_{\mathfrak{g}}^{r}\left(D_{\bullet}\right)\right)_{[k]}=H_{j-1}\left(D_{\bullet}\right)_{[k]}=0$ whenever $j>k+1$. On the other hand, if $j<k$, then $H_{j}\left({ }_{k} \mathcal{Z}_{\bullet}^{+}\right):=H_{j}\left(D_{\bullet}\right)_{[k]}=H_{j+r}\left(H_{\mathfrak{g}}^{r}\left(D_{\bullet}\right)_{[k]}\right)$. However, the latter is zero since end $\left(H_{\mathfrak{g}}^{r}\left(D_{r+j}\right)\right) \leq j$. This shows that ${ }_{k} \mathcal{Z}_{\bullet}^{+}$is exact on the left and also on the right hand of $\tau_{k}$.

It remains to prove the exactness in the joint points $k$ and $k+1$. For this, notice that $\left({ }^{\infty} \mathrm{E}_{\text {hor }}^{-i,-j}\right)_{[k]}=0$ for $j \geq 1$, hence $\mathcal{F}_{j k} / \mathcal{F}_{(j+1) k}=\left({ }^{\infty} \mathrm{E}_{\text {hor }}^{-k-j,-j}\right)_{[k]}=0$ for $j \geq 1$. Consequently, $\mathcal{F}_{1 k}=0$ in (2-6). Therefore, the map $\tau_{k}$ defined in (2-7) is exactly the canonical map $H_{\mathfrak{g}}^{r}\left(D_{r+k}\right)_{[k]} \stackrel{\text { Can. }}{\longrightarrow} \operatorname{Coker}\left(\phi_{k}\right)_{[k]}$ as required.

As already mentioned in the Introduction, some sliding depth conditions are needed to prove the acyclicity of the ${ }_{k} \mathcal{Z}_{\text {. }}^{+}$complexes.

Definition 2.3. Let $(R, \mathfrak{m})$ be a Noetherian local ring of dimension $d$ and let $I=\left(f_{1}, \ldots, f_{r}\right)$ be an ideal of grade $g \geq 1$. Let $k$ and $t$ be two integers. We say that the ideal $I$ satisfies $\mathrm{SD}_{k}$ at level $t$ if $\operatorname{depth}\left(H_{i}(f ; R)\right) \geq \min \{d-g, d-r+i+k\}$ for all $i \geq r-g-t$. Whenever $t=r-g$, we simply say that $I$ satisfies $\mathrm{SD}_{k}$ and we let $\mathrm{SD}$ stand for $\mathrm{SD}_{0}$.

$I$ is strongly Cohen-Macaulay, or SCM, if $H_{i}(f ; R)$ is CM for all $i$. Clearly $\mathrm{SCM}$ is equivalent to $\mathrm{SD}_{r-g}$.

Similarly, we say that $I$ satisfies the sliding depth condition on cycles, or $\mathrm{SDC}_{k}$, at level $t$ if $\operatorname{depth}\left(Z_{i}(f, R)\right) \geq \min \{d-r+i+k, d-g+2, d\}$ for all $i \geq r-g-t$. Again if $t=r-g$, we simply say that $I$ satisfies $\mathrm{SDC}_{k}$ and we use SDC instead of $\mathrm{SDC}_{0}$.

Some of the basic properties and relations between conditions $\mathrm{SD}_{k}$ and $\mathrm{SDC}_{k}$ are explained in the following proposition.

Proposition 2.4. Let $(R, \mathfrak{m})$ be a $C M$ local ring of dimension $d$ and let $I=$ $\left(f_{1}, \ldots, f_{r}\right)$ be an ideal of grade $g \geq 1$. Let $k$ and $t$ be two integers. Then:

(1) The properties $\mathrm{SDC}_{k}$ and $\mathrm{SD}_{k}$ at level tocalize; they only depend on I and not the generating set, if $t=r-g$.

(2) $\mathrm{SD}_{k}$ implies $\mathrm{SDC}_{k+1}$.

(3) $\mathrm{SD}_{k}$ at level $t<r-g$ implies $\mathrm{SDC}_{a}$ at level t for $a \leq g+t+2-d$.

(4) $\mathrm{SDC}_{k+1}$ at level $t$ implies $\mathrm{SD}_{k}$ at level $t$ for any $t$, if $g \geq 2$. This implication is also the case if $g=1$ and $k=0$.

(5) $\mathrm{SD}_{0}$ at level $t \geq 1$ implies $\mathrm{SDC}_{0}$ at level $t$.

Proof. Part (1) is essentially proved in [Vasconcelos 1994]. Part (2) was proved in [Hassanzadeh 2012, Proposition 2.5]. Part (3) follows after analyzing the spectral 
sequences derived from the tensor product of the truncated Koszul complex

$$
0 \rightarrow Z_{i}(f) \rightarrow K_{i} \rightarrow K_{i-1} \rightarrow \cdots \rightarrow K_{0} \rightarrow 0
$$

and the Čech complex $\mathcal{C}_{\mathfrak{m}}^{\bullet}(R)$. To prove (4), we consider the depth inequalities derived from the short exact sequences $0 \rightarrow Z_{i+1}(f) \rightarrow K_{i+1} \rightarrow B_{i}(f) \rightarrow 0$ and $0 \rightarrow B_{i}(f) \rightarrow Z_{i}(f) \rightarrow H_{i}(f) \rightarrow 0$. To show (5), we use the fact that $\mathrm{SDC}_{k}$ holds for any $k$ at level -1 , then a recursive induction applying $\operatorname{depth}\left(Z_{i}\right) \geq$ $\min \left\{\operatorname{depth}\left(H_{i}\right), d, \operatorname{depth}\left(Z_{i+1}\right)-1\right\}$ proves the assertion.

In the next theorem we present sufficient conditions for the acyclicity of ${ }_{k} \mathcal{Z}_{.}^{+}$. The strategy taken here to prove the acyclicity is the same as the one applied in [Hassanzadeh 2012] (however, the reader should notice that the complex $\mathcal{C}$. defined in [Hassanzadeh 2012] is a little bit different from ${ }_{0} \mathcal{Z}_{\text {. }}^{+}$here; in the former, the tail is substituted by a free complex but still $\mathcal{C}$. remains quasi-isomorphic to $\mathcal{Z}^{+}$.). Since the complex is finite, we avail ourselves of "lemme d'aciclicité" of Peskine and Szpiro: we assume some sliding depth conditions and prove the acyclicity in height $s-1$ wherein $I=\mathfrak{a}$. By Lemma $2.2,{ }_{k} \mathcal{Z}^{+}$is exact, if $I=\mathfrak{a}$. Then an induction will show the acyclicity globally.

Although the proof here is more involved than [Hassanzadeh 2012, Proposition 2.8], we prefer to omit it to go faster to newer theorems. The Cohen-Macaulay hypothesis in this theorem is needed to show that if for an $R$-module $M$ we have $\operatorname{depth}(M) \geq d-t$, then for any prime $\mathfrak{p}$ we have $\operatorname{depth}\left(M_{\mathfrak{p}}\right) \geq \operatorname{ht}(\mathfrak{p})-t$ [Vasconcelos 1994, Section 3.3].

Proposition 2.5. Let $(R, \mathfrak{m})$ be a $C M$ local ring of dimension d and let $J=(\mathfrak{a}: I)$ be an $s$-residual intersection. Assume that $I=\left(f_{1}, \ldots, f_{r}\right)$ and $\mathrm{ht}(I)=g \geq 1$. Fix $0 \leq k \leq \max \{s, s-g+2\}$. Then the complex ${ }_{k} \mathcal{Z}^{+}$is acyclic, if any of the following hypotheses holds:

(1) $1 \leq s \leq 2$ and $s=k$, or

(2) $r+k \geq s+1, k \leq 2$, and I satisfies $\mathrm{SDC}_{1}$ at level $s-g-k$, or

(3) $r+k \leq s$ and I satisfies SD, or

(4) $r+k \geq s+1, k \geq 3, I$ satisfies $\operatorname{SDC}_{1}$ at level $s-g-k$, and $\operatorname{depth}\left(Z_{i}(f)\right) \geq$ $d-s+k$ for $0 \leq i \leq k$.

Consequently, having a finite acyclic complex whose components have sufficient depth, one can estimate the depth of the zeroth homology.

Theorem 2.6. Let $(R, \mathfrak{m})$ be a $C M$ local ring of dimension d and let $I=\left(f_{1}, \ldots, f_{r}\right)$ be an ideal with $\mathrm{ht}(I)=g \geq 1$. Let $s \geq g$ and fix $0 \leq k \leq \min \{s, s-g+2\}$. Suppose that one the following hypotheses holds:

(i) $r+k \leq s$ and I satisfies SD, or 
(ii) $r+k \geq s+1$, I satisfies $\mathrm{SDC}_{1}$ at level $s-g-k$, and $\operatorname{depth}\left(Z_{i}(f)\right) \geq d-s+k$ for $0 \leq i \leq k$, or

(iii) $k \leq s-r+2$ and I satisfies $\mathrm{SD}$, or

(iv) $\operatorname{depth}\left(H_{i}(\boldsymbol{f})\right) \geq \min \{d-s+k-2, d-g\}$ for $0 \leq i \leq k-1$ and I satisfies $\mathrm{SD}$, or

(v) I is strongly Cohen-Macaulay.

Then for any s-residual intersection $J=(\mathfrak{a}: I)$, the complex ${ }_{k} \mathcal{Z}_{\bullet}^{+}$is acyclic. Furthermore, $\operatorname{Sym}_{R}^{k}(I / \mathfrak{a})$ if $1 \leq k \leq s-g+2$, or the disguised residual intersection if $k=0$, is CM of codimension $s$.

In the above theorem, the cases where $k=0,1$ and $s-g+1$ are the most important ones. For, $k=0$ is related to the disguised residual intersection, $k=1$ is connected to $R / \mathfrak{a}$, and $k=s-g+1$ is related to the canonical module of $R / J$.

Remark 2.7. Any of the conditions (iii)-(v) are stronger than (i) and (ii). Condition (i) in Theorem 2.6 implies that $\operatorname{depth}(R / I) \geq d-s+k$ and condition (v) implies that depth $(R / I) \geq d-g \geq d-s$. The other conditions, (ii), (iii) and (iv), yield depth $(R / I) \geq d-s+k-2$. Therefore, to have a better consequence of the theorem, it may not be too much to require $\operatorname{depth}(R / I) \geq d-s$.

The first two items in the next corollary settle conjectures (3) and (4) mentioned in the Introduction.

Corollary 2.8. Let $(R, \mathfrak{m})$ be a CM local ring of dimension d and let $I=\left(f_{1}, \ldots, f_{r}\right)$ be an ideal with $\mathrm{ht}(I)=g \geq 1$. Suppose that $\operatorname{depth}(R / I) \geq d-s$ and that $I$ satisfies any of the conditions in Theorem 2.6 for $k=1$, for instance, if I satisfies $S D$. Then, for any algebraic s-residual intersection $J=(\mathfrak{a}: I)$ :

(1) $\operatorname{depth}(R / \mathfrak{a})=d-s$ and thus $\mathfrak{a}$ is minimally generated by s elements.

(2) $J$ is unmixed of height $s$.

(3) $\operatorname{Ass}(R / \mathfrak{a}) \subseteq \operatorname{Ass}(R / I) \cup \operatorname{Ass}(R / J)$. Furthermore, if I is unmixed then the equality holds.

(4) If the residual is arithmetic, then $\operatorname{Sym}_{R}^{i}(I / \mathfrak{a})$ is a faithful $R / J$-module for all $i$.

Proof. (1) Applying Theorem 2.6 for $k=1$, we have $\operatorname{depth}(I / \mathfrak{a})=d-s$. Therefore, the result follows from the exact sequence $0 \rightarrow I / \mathfrak{a} \rightarrow R / \mathfrak{a} \rightarrow R / I \rightarrow 0$ and the fact that $\operatorname{depth}(R / I) \geq d-s$ by hypothesis.

(2) $J$ has codimension at least $s$, thus for any $\mathfrak{p} \in \operatorname{Ass}(R / J)$ we have $h t(\mathfrak{p}) \geq s$. On the other hand, $\operatorname{Ass}(R / J) \subseteq \operatorname{Ass}(R / \mathfrak{a})$ and any prime ideal in the latter has codimension less than $s$ by (1) (see [Bruns and Herzog 1998, Proposition 1.2.13]). This yields the unmixedness of $J$. 
(3) Let $\mathfrak{p} \in \operatorname{Ass}(R / \mathfrak{a})$. Then $\operatorname{ht}(\mathfrak{p}) \leq s$, by (1). If ht $(\mathfrak{p})<s$ or $\mathfrak{p} \not \supset J$, then $\mathfrak{a}_{\mathfrak{p}}=I_{\mathfrak{p}}$ and thus $\mathfrak{p} \in \operatorname{Ass}(R / I)$; otherwise $h t(\mathfrak{p})=s$ and $\mathfrak{p} \supseteq J$, which clearly means $\mathfrak{p} \in \operatorname{Ass}(R / J)$. Verification of the last statement is straightforward.

(4) To see this, we refer to the proof of [Hassanzadeh 2012, Theorem 2.11] wherein it is shown that, in the spectral sequence defined in (2-3), $K:=\left({ }^{\infty} \mathrm{E}_{\text {hor }}^{0,0}\right)_{[0]} \subseteq J \subseteq$ $\left({ }^{2} \mathrm{E}_{\text {hor }}^{0,0}\right)_{[0]}$. Moreover, in the case where the residual is arithmetic, this spectral sequence, locally in height $s$, collapses in the second page. Hence all of these inclusions are equality. We just notice that

$$
\left({ }^{2} \mathrm{E}_{\text {hor }}^{0,0}\right)_{[0]}=H_{\mathfrak{g}}^{0}\left(H_{0}\left(D_{\bullet}\right)\right)_{[0]}=\bigcup_{i=0}^{\infty}\left(\boldsymbol{\gamma} \cdot \operatorname{Sym}_{R}^{i}(I):_{R} \operatorname{Sym}_{R}^{i+1}(I)\right) .
$$

Thus once equality in the above line happens, $J=\boldsymbol{\gamma} \cdot \operatorname{Sym}_{R}^{i}(I):_{R} \operatorname{Sym}_{R}^{i+1}(I)$ for all $i$. Therefore, $\operatorname{Sym}_{R}^{i}(I / \mathfrak{a})=\operatorname{Sym}_{R}^{i+1}(I) / \boldsymbol{\gamma} \cdot \operatorname{Sym}_{R}^{i}(I)$ is a faithful $R / J$-module.

The following examples show the accuracy of the conditions in Theorem 2.6 and Corollary 2.8(4).

Example 2.9. Let

$$
R=\mathbb{Q}\left[x_{1}, \ldots, x_{7}\right], \quad M=\left(\begin{array}{cccc}
0 & x_{1} & x_{2} & x_{3} \\
x_{4} & x_{5} & x_{6} & x_{7}
\end{array}\right), \quad \text { and } \quad I=I_{2}(M) .
$$

Set

$$
\begin{array}{ll}
f_{0}=-x_{4} x_{1}, & f_{3}=-x_{3} x_{4}, \\
f_{1}=-x_{2} x_{4}, & f_{4}=x_{1} x_{7}-x_{3} x_{5}, \\
f_{2}=x_{1} x_{6}-x_{2} x_{5}, & f_{5}=x_{2} x_{7}-x_{3} x_{6} .
\end{array}
$$

Macaulay2 computations show that $\operatorname{depth}\left(Z_{1}(I)\right)=6, \operatorname{depth}\left(Z_{2}(I)\right)=2$ and $\operatorname{depth}\left(Z_{3}(I)\right)=6$, hence $I$ satisfies $\mathrm{SDC}_{1}$ at level 0 but not at level 1 . However, $Z_{1}$ has enough depth, hence condition (ii) in Theorem 2.6 is fulfilled whenever $2 \leq s \leq 4$. The nontrivial case will be $s=4$. Also, $\operatorname{depth}(R / I)=4>d-s=3$. It then follows from Corollary 2.8 that for any 4-residual intersection $J=\mathfrak{a}: I$ we have $\operatorname{ht}(J)=4=\mu(\mathfrak{a})$. It is easy to see that $I$ satisfies $G_{\infty}$; hence [Eisenbud et al. 2004, Proposition 3.7bis] implies that $\ell(I) \geq 5$. On the other hand, the Plüker relations show that $f_{2} f_{3}-f_{1} f_{4}+f_{0} f_{5}=0$. Hence $I$ is not generated by analytically independent elements; that is, $\ell(I)=5$. Therefore, the same proposition in [loc. cit.] proves that there must exist a 5-residual intersection $J=\mathfrak{a}: I$ such that $h t(J)>5$. By the latter, Corollary 2.8 guarantees that, for that ideal $\mathfrak{a}$, the module $I / \mathfrak{a}$ cannot be $\mathrm{CM}$ and thus Theorem 2.6 does not work because $\mathrm{SDC}_{1}$ at level 1 is not satisfied.

Another note about this example is that $\operatorname{depth}\left(R / I^{2}\right)=2$, according to Macaulay2; therefore, $\operatorname{Ext}^{5}\left(R / I^{2}, R\right) \neq 0$. The vanishing of this Ext module would be sufficient 
to prove that $I$ is 4-residually $S_{2}$ in [Chardin et al. 2001, Theorem 4.1], whereas we saw in the above corollary that $I$ is 4-residually unmixed.

Example 2.10. The arithmetic hypothesis in Corollary 2.8(4) cannot be dropped. Let $R=\mathbb{Q}[x, y], I=(x, y)$ and $\mathfrak{a}=\left(x^{2}, y^{2}\right)$. Then we have $J=(\mathfrak{a}, x y)$ and $\operatorname{Ann}\left(\operatorname{Sym}_{R}^{2}(I / \mathfrak{a})\right)=\left(\mathfrak{a} I: I^{2}\right)=(x, y)$. The latter is not $J$, since the residual (linkage) is not arithmetic.

In the case where the residual intersection is geometric, we have stronger corollaries.

Corollary 2.11. Let $(R, \mathfrak{m})$ be a $C M$ local ring of dimension $d$ and let $I=$ $\left(f_{1}, \ldots, f_{r}\right)$ be an ideal with $\mathrm{ht}(I)=g \geq 1$. Suppose that $\operatorname{depth}(R / I) \geq d-s$ and that I satisfies any of the conditions in Theorem 2.6 for some $1 \leq k \leq s-g+2$. Then, for any geometric $s$-residual intersection $J=(\mathfrak{a}: I)$ and for that $k$ :

(1) $I^{k} / \mathfrak{a} I^{k-1}$ is isomorphic to $\operatorname{Sym}_{R}^{k}(I / \mathfrak{a})$ and it is a faithful maximal CohenMacaulay $R / J$-module.

(2) $\mathfrak{a} I^{k-1}=I^{k} \cap J$.

(3) $I^{k}+J$ is a CM ideal of height $s+1$ and $\operatorname{ht}\left(I^{k}+J / J\right)=1$.

Proof.

(1) Consider the natural map

$$
\varphi: \frac{\operatorname{Sym}_{R}^{k}(I)}{\gamma \operatorname{Sym}_{R}^{k-1}(I)} \rightarrow \frac{I^{k}}{\mathfrak{a} I^{k-1}}
$$

that sends $T_{i}$ to $f_{i}$. The map $\varphi$ is onto. Let $K=\operatorname{Ker}(\varphi)$. We show that $\operatorname{Ass}(K)=\varnothing$, hence $K=0$. We have

$$
\operatorname{Ass}(K) \subseteq \operatorname{Ass}\left(\frac{\operatorname{Sym}_{R}^{k}(I)}{\gamma \operatorname{Sym}_{R}^{k-1}(I)}\right) .
$$

By Theorem 2.6,

$$
\operatorname{depth}\left(\frac{\operatorname{Sym}_{R}^{k}(I)}{\gamma \operatorname{Sym}_{R}^{k-1}(I)}\right)=d-s,
$$

hence for any associated prime $\mathfrak{p}$ in the latter we have ht $(\mathfrak{p}) \leq s$. However, since $J$ is a geometric residual, for any $\mathfrak{p}$ of codimension $\leq s$, either $I_{\mathfrak{p}}=\mathfrak{a}_{\mathfrak{p}}$ or $I_{\mathfrak{p}}=(1)$. In the former case,

$$
\frac{\operatorname{Sym}_{R}^{k}\left(I_{\mathfrak{p}}\right)}{\gamma_{\mathfrak{p}} \operatorname{Sym}_{R}^{k-1}\left(I_{\mathfrak{p}}\right)}=0
$$

for $k \geq 1$, which implies that $\mathfrak{p} \notin \operatorname{Ass}(K)$. In the latter case, $\operatorname{Sym}_{R}^{k}\left(I_{\mathfrak{p}}\right)=R_{\mathfrak{p}}$, hence $\varphi_{\mathfrak{p}}$ is an isomorphism, which means $K_{\mathfrak{p}}=0$; in particular, $\mathfrak{p} \notin \operatorname{Ass}(K)$. Therefore, $\operatorname{Ass}(K)=\varnothing$, hence $K=0$. The CM-ness of $\operatorname{Sym}_{R}^{k}(I / \mathfrak{a})$ was already shown in 
Theorem 2.6. To see that $I^{k} / \mathfrak{a} I^{k-1}$ is faithful, one may use Corollary 2.8 and part (1), or just notice that $J \subseteq \mathfrak{a} I^{k-1}: I^{k}$ and that the equality holds locally at all associated primes of $J$ which are of height $s$.

(2) Consider the canonical map $\psi: I^{k} /\left(\mathfrak{a} I^{k-1}\right) \rightarrow\left(I^{k}+J\right) / J$. By (2), $I^{k} / \mathfrak{a} I^{k-1}$ is $\mathrm{CM}$ of dimension $d-s$, which in conjunction with the fact that the residual is geometric shows that $\psi$ is an isomorphism for all $0 \leq k \leq s-g+2$. Notice that $\left(I^{k}+J\right) / J \simeq I^{k} /\left(J \cap I^{k}\right)$. Thus the canonical map implies $J \cap I^{k}=\mathfrak{a} I^{k-1}$.

(3) By (1) and (2), $\left(I^{k}+J\right) / J$ is CM of dimension $d-s$. Also, by Theorem 2.6, $R / J$ is $\mathrm{CM}$ of dimension $d-s$. Thus the exact sequence $0 \rightarrow\left(I^{k}+J\right) / J \rightarrow$ $R / J \rightarrow R /\left(I^{k}+J\right) \rightarrow 0$ implies $\operatorname{depth}\left(R / I^{k}+J\right) \geq d-s-1$. On the other hand, since the residual is geometric, $\operatorname{ht}\left(I^{k}+J\right) \geq s+1$, which in turn completes the proof. To see that ht $\left(\left(I^{k}+J\right) / J\right)=1$, it is enough to notice that $R / J$ is CM.

We now proceed to determine the structure of the canonical module of $R / J$. This achievement is via a study of the type of an appropriate candidate for the canonical module. Recall that in a Noetherian local ring $(R, \mathfrak{m})$ the type of a finitely generated module $M$ is the dimension of the $R / \mathfrak{m}$-vector space $\operatorname{Ext}_{R}^{\operatorname{depth}(M)}(R / \mathfrak{m}, M)$, and it is denoted by $r_{R}(M)$ or just $r(M)$.

Theorem 2.12. Let $(R, \mathfrak{m})$ be a CM local ring of dimension d and let $I=\left(f_{1}, \ldots, f_{r}\right)$ be an ideal with $\mathrm{ht}(I)=g \geq 2$. Let $J=(\mathfrak{a}: I)$ be an s-residual intersection of $I$ and let $1 \leq k \leq s-g+1$. Suppose that I is SCM. Then

$$
r_{R}\left(\operatorname{Sym}_{R}^{k}(I / \mathfrak{a})\right) \leq\left(\begin{array}{c}
r+s-g-k \\
r-1
\end{array}\right) r_{R}(R) .
$$

Proof. First we deal with the case where $g=2$. Consider the $\operatorname{complex}_{k} \mathcal{Z}_{\bullet}^{+}$,

$$
\begin{aligned}
0 \rightarrow H_{\mathfrak{g}}^{r}\left(D_{r+s-1}\right)_{[k]} \rightarrow \cdots \stackrel{\phi_{k}}{\longrightarrow} H_{\mathfrak{g}}^{r}\left(D_{r+k}\right)_{[k]} \\
\qquad \stackrel{\tau_{k}}{\longrightarrow}\left(D_{k}\right)_{[k]} \rightarrow \cdots \rightarrow\left(D_{0}\right)_{[k]} \rightarrow 0 .
\end{aligned}
$$

We study the depth of the components of this complex. Since $Z_{r-1}=R$, we have $\operatorname{depth}\left({ }_{k} \mathcal{Z}_{r+s-1}^{+}\right)=d$. For $k \leq j \leq s-2$, the first cycle which appears in $D_{r+j}$ is $Z_{r-s+j}$ and, by the SCM condition, it has depth equal to $d=d-g+2$, which is at least $d-s+(j+1)+1$. As for the right part of the complex, we have $\operatorname{depth}\left(Z_{i}\right)=d-g+2 \geq d-s+k+1$ for all $0 \leq i \leq k$, since by assumption $k \leq s-g+1$.

Summing up, we have

$$
\operatorname{depth}\left({ }_{k} \mathcal{Z}_{i}^{+}\right) \geq d-s+i+1 \text { for all } i=0, \ldots, s-1 .
$$

Now, let $F_{.} \rightarrow R / \mathfrak{m}$ be a free resolution of $R / \mathfrak{m}$. We put the double complex $\operatorname{Hom}_{R}\left(F_{\bullet},{ }_{k} \mathcal{Z}_{\bullet}^{+}\right)$in the third quadrant with $\operatorname{Hom}_{R}\left(F_{0},{ }_{k} \mathcal{Z}_{0}^{+}\right)$in the center. The two 
arisen spectral sequences are

${ }^{\infty} \mathrm{E}_{\mathrm{hor}}^{-i,-j}=\left\{\begin{array}{ll}\operatorname{Ext}_{R}^{j}\left(R / \mathfrak{m}, \operatorname{Sym}_{R}^{k}(I / \mathfrak{a})\right), & i=0, \\ 0, & i \neq 0,\end{array}\right.$ and ${ }^{1} \mathrm{E}_{\mathrm{ver}}^{-i,-j}=\operatorname{Ext}_{R}^{j}\left(R / \mathfrak{m},{ }_{k} \mathcal{Z}_{i}^{+}\right)$.

The latter vanishes for all $(i, j)$ such that $j-i \leq d-s$ and $i \neq s$, according to (2-12). The convergence of both complexes to the homology module of the total complex implies that $\operatorname{Ext}_{R}^{d-s}\left(R / \mathfrak{m}, \operatorname{Sym}_{R}^{k}(I / \mathfrak{a})\right) \simeq{ }^{\infty} \mathrm{E}_{\mathrm{ver}}^{-s,-d}$. The latter is a submodule of $\operatorname{Ext}_{R}^{d}\left(R / \mathfrak{m},{ }_{k} \mathcal{Z}_{s}^{+}\right)$. Therefore, $r_{R}\left(\operatorname{Sym}_{R}^{k}(I / \mathfrak{a})\right) \leq \operatorname{dim}_{R / \mathfrak{m}}\left(\operatorname{Ext}_{R}^{d}\left(R / \mathfrak{m},{ }_{k} \mathcal{Z}_{s}^{+}\right)\right)$.

Following from the construction of $\mathcal{D}_{\bullet}$, we have $D_{r+s-1}=S(-r+1-s)$. Hence

$$
{ }_{k} \mathcal{Z}_{s}^{+}=H_{\mathfrak{g}}^{r}(S)_{[-r+1-s+k]} .
$$

To calculate the dimension of the above Ext module, we just need to know how many copies of $R$ appear in the inverse polynomial structure of the above local cohomology module. The answer is simply the number of positive solutions of the numerical equation $\alpha_{1}+\cdots+\alpha_{r}=r-1+s-k$, which is $\left(\begin{array}{c}r-k+s-2 \\ r-1\end{array}\right)$. Therefore, $r_{R}\left(\operatorname{Sym}_{R}^{k}(I / \mathfrak{a})\right) \leq\left(\begin{array}{c}r+s-2-k \\ r-1\end{array}\right) r(R)$ in the case where $g=2$.

Now, let ht $(I)=g \geq 2$. We choose a regular sequence $\boldsymbol{a}$ of length $g-2$ inside $\mathfrak{a}$ which is a part of a minimal generating set of $\mathfrak{a} . I / \boldsymbol{a}$ is still SCM by [Huneke 1983, Corollary 1.5]; moreover, $J / \boldsymbol{a}=\mathfrak{a} / \boldsymbol{a}: I / \boldsymbol{a}$. Recall that $\mu(\mathfrak{a})=s$ by Corollary 2.8; therefore, $J / \boldsymbol{a}$ is an $(s-g+2)$-residual intersection of $I / \boldsymbol{a}$ which is of height 2. Hence we return to the case $g=2$ to obtain

$$
\begin{aligned}
r_{R}\left(\operatorname{Sym}_{R}^{k}(I / \mathfrak{a})\right) & =r_{R / \boldsymbol{a}}\left(\operatorname{Sym}_{R / \boldsymbol{a}}^{k}(I / \mathfrak{a})\right) \\
& \leq\left(\begin{array}{c}
r+(s-g+2)-2-k \\
r-1
\end{array}\right) r_{R / \boldsymbol{a}}(R / \boldsymbol{a})=\left(\begin{array}{c}
r+s-g-k \\
r-1
\end{array}\right) r_{R}(R)
\end{aligned}
$$

(see [Bruns and Herzog 1998, Exercise 1.2.26]).

Remark 2.13. Clearly the above proof works for $k=0$ as well; hence if $I$ is SCM of $h t(I) \geq 2$ and $K$ is a disguised $s$-residual intersection of $I$, then

$$
r_{R}(R / K) \leq\left(\begin{array}{c}
r+s-g \\
r-1
\end{array}\right) r_{R}(R) .
$$

Everything is now ready for us to explain the structure of the canonical module. Theorem 2.14. Suppose that $(R, \mathfrak{m})$ is a Gorenstein local ring of dimension d. Let $I$ be an SCM ideal with $\mathrm{ht}(I)=g$ and let $J=(\mathfrak{a}: I)$ be an arithmetic s-residual intersection of I. Then $\omega_{R / J}$ is isomorphic to $\operatorname{Sym}_{R}^{s-g+1}(I / \mathfrak{a})$. It is isomorphic to $\left(I^{s-g+1}+J\right) / J$ in the geometric case. Furthermore, $R / J$ is generically Gorenstein and $R / J$ is Gorenstein if and only if $I / \mathfrak{a}$ is principal.

Proof. Without loss of generality, we may suppose that $g \geq 2$; otherwise we may add a new variable $x$ to $R$ and consider ideals $\mathfrak{a}+(x)$ and $I+(x)$ in the local ring $R[x]_{\mathfrak{m}+(x)}$ wherein we have $J+(x)=(\mathfrak{a}+(x)):(I+(x))$. 
$R / J$ is CM by Theorem 2.6. Respectively, by Theorem 2.6, Corollary 2.8 and Theorem 2.12, $\operatorname{Sym}_{R}^{s-g+1}(I / \mathfrak{a})$ is a maximal CM, faithful $R / J$-module and of type 1. Therefore, it is the canonical module of $R / J$ by [Bruns and Herzog 1998, Proposition 3.3.13]. In the geometric case, $\operatorname{Sym}_{R}^{s-g+1}(I / \mathfrak{a}) \simeq\left(I^{s-g+1}+J\right) / J$ by Corollary 2.11 .

To see that $R / J$ is generically Gorenstein, notice that for any prime ideal $\mathfrak{p} \supseteq J$ of height $s$ we have $r\left((R / J)_{\mathfrak{p}}\right)=\mu\left(\left(\omega_{R / J}\right)_{\mathfrak{p}}\right)=\mu\left(\operatorname{Sym}_{R_{\mathfrak{p}}}^{s-g+1}\left((I / \mathfrak{a})_{\mathfrak{p}}\right)\right)$, which is 1 since the residual is arithmetic by the assumption. Finally, let $t=\mu(I / \mathfrak{a})$; then $r(R / J)=\mu\left(\omega_{R / J}\right)=\left(\begin{array}{c}s-g+t \\ t-1\end{array}\right)$ which is 1 if and only if $t=1$.

\section{Hilbert function, Castelnuovo-Mumford regularity}

Although the family $\left\{{ }_{k} \mathcal{Z}_{\text {. }}^{+}\right\}$does not consist of free complexes, it can approximate $R / J$ and $\operatorname{Sym}_{R}^{k}(I / \mathfrak{a})$ very closely. Several numerical invariants or functions such as Castelnuovo-Mumford regularity, projective dimension and the Hilbert function can be estimated via this family. Chardin, Eisenbud and Ulrich [Chardin et al. 2001] restated an old question [Stanley 1980] asking for which open sets of ideals $\mathfrak{a}$ the Hilbert function of $R / \mathfrak{a}$ depends only on the degrees of the generators of $\mathfrak{a}$. In [Chardin et al. 2001], the authors consider the following two conditions:

(A1) if the Hilbert function of $R / \mathfrak{a}$ is constant on the open set of ideals $\mathfrak{a}$ generated by $s$ forms of the given degrees such that $\operatorname{codim}(\mathfrak{a}: I) \geq s$; and

(A2) if the Hilbert function of $R /(\mathfrak{a}: I)$ is constant on this set.

It is shown in [Chardin et al. 2001, Theorem 2.1] that ideals with some slight depth conditions in conjunction with $G_{s-1}$ or $G_{s}$ satisfy these two conditions. In this direction we have the following result which, in addition to showing the validity of conditions (A1) and (A2) under some sliding depth condition, provides a method for computing the desired Hilbert functions.

Proposition 3.1. Let $R$ be a $C M$ graded ring over an Artinian local ring $R_{0}$, and let $I$ and $\mathfrak{a}$ be two homogeneous ideals of $R$. Let $J=(\mathfrak{a}: I)$ be an s-residual intersection. Let $0 \leq k \leq s-g+2$ and suppose that I satisfies any of the following hypotheses:

(1) $1 \leq s \leq 2$ and $s=k$, or

(2) $r+k \geq s+1, k \leq 2$, and I satisfies $\mathrm{SDC}_{1}$ at level $s-g-k$, or

(3) $r+k \leq s$ and I satisfies SD, or

(4) $r+k \geq s+1, k \geq 3, I$ satisfies $\operatorname{SDC}_{1}$ at level $s-g-k$, and $\operatorname{depth}\left(Z_{i}(f)\right) \geq$ $d-s+k$ for $0 \leq i \leq k$.

Then the Hilbert function of the disguised s-residual intersection, if $k=0$, and that of $\operatorname{Sym}_{R}^{k}(I / \mathfrak{a})$, if $1 \leq k \leq s-g+2$, depends only on the degrees of the generators 
of $\mathfrak{a}$ and the Koszul homologies of I. In particular, if I satisfies any of the above conditions for $k=1$ then the Hilbert function of $R / \mathfrak{a}$ satisfies (A1).

Proof. By Proposition 2.5, the standing assumptions on $I$ imply the acyclicity of ${ }_{k} \mathcal{Z}_{\text {. }}^{+}$. Hence the Hilbert function of $H_{0}\left({ }_{k} \mathcal{Z}_{\bullet}^{+}\right)$is derived from the Hilbert functions of the components of ${ }_{k} \mathcal{Z}^{+}$which, according to (2-1) and (2-9), are just some direct sums of the Koszul cycles of $I$ shifted by the twists appearing in the Koszul complex $K .(\gamma, S)$. Since the Hilbert functions of Koszul cycles are inductively calculated in terms of those of the Koszul homologies, the Hilbert function of $H_{0}\left({ }_{k} \mathcal{Z}_{\bullet}^{+}\right)$depends on the Koszul homologies of $I$ and just the degrees of the generators of $\mathfrak{a}$. In the case where $k=1$, we know the Hilbert function of $I / \mathfrak{a}=\operatorname{Sym}_{R}^{1}(I / \mathfrak{a})$ and therefore we know the Hilbert function of $R / \mathfrak{a}$ by $0 \rightarrow I / \mathfrak{a} \rightarrow R / \mathfrak{a} \rightarrow R / I \rightarrow 0$.

The next important numerical invariant associated to an algebraic or geometric object is Castelnuovo-Mumford regularity. Here we present an upper bound for the regularity of the disguised $s$-residual intersection $K$ and the regularity of $\operatorname{Sym}_{R}^{k}(I / \mathfrak{a})$ (if $1 \leq k \leq s-g+1$ ).

Assume that $R=\bigoplus_{n \geq 0} R_{n}$ is a positively graded *local Noetherian ring of dimension $d$ over a Noetherian local ring $\left(R_{0}, \mathfrak{m}_{0}\right)$, and set $\mathfrak{m}=\mathfrak{m}_{0}+R_{+}$. Suppose that $I$ and $\mathfrak{a}$ are homogeneous ideals of $R$ generated by homogeneous elements $f_{1}, \ldots, f_{r}$ and $a_{1}, \ldots, a_{s}$, respectively. Let $\operatorname{deg} f_{t}=i_{t}$ for all $1 \leq t \leq r$ with $i_{1} \geq \cdots \geq i_{r}$ and $\operatorname{deg} a_{t}=l_{t}$ for $1 \leq t \leq s$. For a graded ideal $\mathfrak{b}$, the sum of the degrees of a minimal generating set of $\mathfrak{b}$ is denoted by $\sigma(\mathfrak{b})$.

Proposition 3.2. With the same notation just introduced above, let $(R, \mathfrak{m})$ be a $C M$ *local ring, let $I$ be an ideal with $\mathrm{ht}(I)=g \geq 2$ and let $s \geq g$. Suppose that $J=(\mathfrak{a}: I)$ is an s-residual intersection of $I$ and $K$ is the disguised s-residual intersection of I with respect to $\mathfrak{a}$. Suppose that any of the following conditions hold for some $0 \leq k \leq s-g+1$ :

(i) $r+k \leq s$ and I satisfies $\mathrm{SD}_{1}$, or

(ii) $r+k \geq s+1$, I satisfies $\mathrm{SDC}_{2}$ at level $s-g-k$, and $\operatorname{depth}\left(Z_{i}(\boldsymbol{f})\right) \geq d-s+k+1$ for $0 \leq i \leq k$.

Then if $k \geq 1$,

$$
\operatorname{reg}\left(\operatorname{Sym}_{R}^{k}(I / \mathfrak{a})\right) \leq \operatorname{reg}(R)+\operatorname{dim}\left(R_{0}\right)+\sigma(\mathfrak{a})-(s-g+1-k) \operatorname{indeg}(I / \mathfrak{a})-s .
$$

And if $k=0$,

$$
\operatorname{reg}(R / K) \leq \operatorname{reg}(R)+\operatorname{dim}\left(R_{0}\right)+\sigma(\mathfrak{a})-(s-g+1) \operatorname{indeg}(I / \mathfrak{a})-s .
$$

Proof. The proof of this fact is essentially the same as that of [Hassanzadeh 2012, Theorem 3.6] wherein the case of $k=0$ is verified. A crucial part of repeating that proof is that we have to change the structure of the families $\left\{{ }_{k} \mathcal{Z}_{\bullet}^{+}\right\}$from the 
beginning by substituting the tails with a free complex. Another way to prove this result in the case where $I$ is SCM may be by applying the " $g=2$ " trick in Theorem 2.12.

The inequality in the above proposition becomes more interesting in particular cases; for example, if $R$ is a polynomial ring over a field and $k=1$, this inequality reads as

$$
\operatorname{reg}_{R}(I / \mathfrak{a})+(s-g) \operatorname{indeg}(I / \mathfrak{a}) \leq \sigma(\mathfrak{a})-s .
$$

Hence we have a numerical obstruction for $J=(\mathfrak{a}: I)$ to be an $s$-residual intersection.

At the other end of the spectrum we have $k=s-g+1$. This case corresponds to the canonical module of $R / J$.

The next proposition improves [Hassanzadeh 2012, Proposition 3.5] by removing the $G_{s}$ condition.

Proposition 3.3. Suppose that $(R, \mathfrak{m})$ is a positively graded Gorenstein *local ring, over a Noetherian local ring $\left(R_{0}, \mathfrak{m}_{0}\right)$, with canonical module $\omega_{R}=R(b)$ for some integer $b$. Let I be a homogeneous SCM ideal with $\mathrm{ht}(I)=g$, let $\mathfrak{a} \subset I$ be homogeneous and let $J=(\mathfrak{a}: I)$ be an arithmetic s-residual intersection of $I$. Then $\omega_{R / J}=\operatorname{Sym}_{R}^{s-g+1}(I / \mathfrak{a})(b+\sigma(\mathfrak{a}))$.

In particular, if $\operatorname{dim}\left(R_{0}\right)=0$ then

$$
\operatorname{reg}(R / J)=\operatorname{reg}(R)+\sigma(\mathfrak{a})-\operatorname{indeg}\left(\operatorname{Sym}_{R}^{s-g+1}(I / \mathfrak{a})\right)-s .
$$

Proof. We may and do assume that $g \geq 2$. First suppose that $g=2$. We keep the notation defined at the beginning of Section 2 to define the complex ${ }_{k} \mathcal{Z}^{+}$. Considering $R$ as a subalgebra of $S=R\left[T_{1}, \ldots, T_{r}\right]$, we write the degrees of an element $x$ of $R$ as the 2-tuple ( $\operatorname{deg} x, 0)$ with the second entry zero. Therefore, $\operatorname{deg} f_{t}=\left(i_{t}, 0\right)$ for all $1 \leq t \leq r, \operatorname{deg} a_{t}=\left(l_{t}, 0\right)$ for all $1 \leq t \leq s, \operatorname{deg} T_{t}=\left(i_{t}, 1\right)$ for all $1 \leq t \leq r$, and thus $\operatorname{deg} \gamma_{t}=\left(l_{t}, 1\right)$ for all $1 \leq t \leq s$. With this notation the $\mathcal{Z}$-complex has the shape

$$
\mathcal{Z}_{.}: 0 \rightarrow Z_{r-1} \otimes_{R} S(0,-r+1) \rightarrow \cdots \rightarrow Z_{1} \otimes_{R} S(0,-1) \rightarrow Z_{0} \otimes_{R} S \rightarrow 0 .
$$

Consequently,

$$
\mathcal{Z}_{r-1}=R\left(-\sum_{t=1}^{r} i_{t}, 0\right) \otimes_{R} S(0,-r+1),
$$

and, taking into account the fact that $a_{1}, \ldots, a_{s}$ is a minimal generating set of $\mathfrak{a}$ by Corollary 2.8,

$$
D_{r+s-1}=S\left(-\sum_{t=1}^{r} i_{t}-\sigma(\mathfrak{a}),-s-r+1\right) .
$$


It then follows that ${ }_{(s-1)} \mathcal{Z}_{s}^{+}=H_{\mathfrak{g}}^{r}\left(D_{r+s-1}\right)_{[*, s-1]}$ is isomorphic to

$$
\begin{aligned}
H_{\mathfrak{g}}^{r}(S)\left(-\sum_{t=1}^{r} i_{t}-\sigma(\mathfrak{a})\right. & , 0)_{[*,-r]} \\
& \simeq\left(R T_{1}^{-1} \cdots T_{r}^{-1}\left(-\sum_{t=1}^{r} i_{t}\right)\right)(-\sigma(\mathfrak{a})) \simeq R(-\sigma(\mathfrak{a})) .
\end{aligned}
$$

Notice that $\operatorname{deg}\left(T_{j}^{-1}\right)=\left(-i_{j},-1\right)$.

We next turn to the proof of Theorem 2.12 and consider the spectral sequences therein for $k=s-g+1=s-1$. We have ${ }^{1} \mathrm{E}_{\mathrm{ver}}^{-s,-d}=\operatorname{Ext}_{R}^{d}\left(R / \mathfrak{m},{ }_{(s-1)} \mathcal{Z}_{s}^{+}\right)$ which is isomorphic to $\operatorname{Ext}_{R}^{d}(R / \mathfrak{m}, R(b))(-b-\sigma(\mathfrak{a}))$ by (3-1). The latter is in turn homogeneously isomorphic to $R / \mathfrak{m}(-b-\sigma(\mathfrak{a}))$, since $R$ is Gorenstein. The inclusion

$\operatorname{Ext}_{R}^{d-s}\left(R / \mathfrak{m}, \operatorname{Sym}_{R}^{s-g+1}(I / \mathfrak{a})\right) \simeq{ }^{\infty} \mathrm{E}_{\mathrm{hor}}^{0,-(d-s)} \hookrightarrow{ }^{1} \mathrm{E}_{\mathrm{ver}}^{-s,-d}=\operatorname{Ext}_{R}^{d}\left(R / \mathfrak{m},(s-1) \mathcal{Z}_{s}^{+}\right)$

is indeed an isomorphism, since the latter is a vector space of dimension one. In the graded case, all of the homomorphisms in the proof of Theorem 2.12 are homogeneous; therefore, the above isomorphism implies $\operatorname{Ext}_{R}^{d-s}\left(R / \mathfrak{m}, \operatorname{Sym}_{R}^{s-g+1}(I / \mathfrak{a})\right) \simeq$ $R / \mathfrak{m}(-b-\sigma(\mathfrak{a}))$. We already know that in the local case $\omega_{R / J}=\operatorname{Sym}_{R}^{s-2+1}(I / \mathfrak{a})$, by Theorem 2.14, hence in the graded case we have $\omega_{R / J}=\operatorname{Sym}_{R}^{s-2+1}(I / \mathfrak{a})(b+\sigma(\mathfrak{a}))$.

Now suppose that $g \geq 2$ and let $\boldsymbol{a}=a_{1}, \ldots, a_{g-2}$ be a regular sequence in $\mathfrak{a}$ which is a part of its minimal generating set. Then $\omega_{R / \boldsymbol{a}}=(R / \boldsymbol{a})(b+\sigma(\boldsymbol{a}))$ and, moreover, we go back to the case where $g=2$. We then have

$$
\begin{aligned}
\omega_{R / J}=\operatorname{Sym}_{R / \boldsymbol{a}}^{(s-g+2)-2+1}\left(\frac{I / \boldsymbol{a}}{\mathfrak{a} / \boldsymbol{a}}\right)((b+\sigma(\boldsymbol{a}))+ & \sigma(\mathfrak{a} / \boldsymbol{a})) \\
& =\operatorname{Sym}_{R}^{s-g+1}(I / \mathfrak{a})(b+\sigma(\mathfrak{a})) .
\end{aligned}
$$

Finally, one may ask whether under the $G_{s}$ condition our methods can contribute more information about the structure of residual intersections than what is known so far. The next result shows that in the presence of the $G_{s}$ condition the complex ${ }_{0} \mathcal{Z}_{\bullet}^{+}$approximates $R / J$ for any (algebraic) $s$-residual intersection $J$. In particular, the above facts about regularity and the Hilbert function hold for $R / J$.

Proposition 3.4. Let $(R, \mathfrak{m})$ be a Gorenstein local ring and suppose that $I$ is $S C M$ and evenly linked to an ideal which satisfies $G_{s}$. Then the disguised s-residual intersections of $I$, with respect to an ideal $\mathfrak{a}$, is the same as the algebraic residual intersection $J=\mathfrak{a}: I$.

Proof. It is shown in [Huneke and Ulrich 1988, Theorem 5.3] that under the above assumptions on $I$ there exists a deformation $\left(R^{\prime}, I^{\prime}\right)$ of $(R, I)$ such that $I^{\prime}$ is $\mathrm{SCM}$ and $G_{s}$. Let $I=\left(f_{1}, \ldots, f_{r}\right), \mathfrak{a}=\left(a_{1}, \ldots, a_{s}\right)$, and suppose that 
$a_{i}=\sum_{j=1}^{r} c_{i j} f_{j}$. Let $s_{i j}$ be a lifting of $c_{i j}$ to $R^{\prime}$ and let $f_{i}^{\prime}$ be a lifting of $f_{i}$ to $R^{\prime}$. Let $X=\left(x_{i j}\right)$ be an $s \times r$ matrix of invariants and define $\alpha:=\left(\alpha_{1}, \ldots, \alpha_{s}\right)=$ $X \cdot\left(f_{1}^{\prime}, \ldots, f_{r}^{\prime}\right)^{t}$. Let $\tilde{R}=R^{\prime}\left[x_{i j}\right]_{\left(\mathfrak{m}+\left(x_{i j}-s_{i j}\right)\right)}$ and $\tilde{J}:=\left(\alpha_{1}, \ldots, \alpha_{s}\right):_{\tilde{R}} I^{\prime} \tilde{R}$. By [Huneke and Ulrich 1988, Lemma 3.2], $\tilde{J}$ is a geometric $s$-residual intersection of $I^{\prime} \tilde{R}$. We construct the complex ${ }_{0} \mathcal{Z}_{\bullet}^{+}\left(\left(\alpha_{1}, \ldots, \alpha_{s}\right) ;\left(f_{1}^{\prime}, \ldots, f_{s}^{\prime}\right)\right)$ in $\tilde{R}$ and set $\tilde{R} / \tilde{K}=H_{0}\left({ }_{0} \mathcal{Z}_{\bullet}^{+}\left(\left(\alpha_{1}, \ldots, \alpha_{s}\right) ;\left(f_{1}^{\prime}, \ldots, f_{s}^{\prime}\right)\right)\right)$. Since $\tilde{J}$ is a geometric residual, we have $\tilde{J}=\tilde{K}$.

Assume that $\pi$ is the projection from $\tilde{R}$ to $R$ which sends $x_{i j}$ to $c_{i j}$. By [Hassanzadeh 2012, Proposition 2.13], $R / \pi(\tilde{K})=H_{0}\left({ }_{0} \mathcal{Z}_{.}^{+}\right)$and, by [Huneke and Ulrich 1988, Theorem 4.7], $\pi(\tilde{J})=J$. Therefore, $R / \pi(\tilde{K})=R / \pi(\tilde{J})=R / J$.

\section{Arithmetic residual intersections}

In this section we scrutinize the structure of the ${ }_{0} \mathcal{Z}^{+}$complex in the case where $I / \mathfrak{a}$ is cyclic. We find that the homologies of ${ }_{0} \mathcal{Z}^{+}$determine the uniform annihilator of nonzero Koszul homologies. The importance of this fact is that, although the Koszul complex is an old object, not much is known about the annihilator of higher homologies. Besides the rigidity and differential graded algebra structure of the homologies, there is an interesting paper of Corso, Huneke, Katz and Vasconcelos [Corso et al. 2006] wherein the authors make efforts to find out "whether the annihilators of nonzero Koszul homology modules of an unmixed ideal is contained in the integral closure of that ideal". However, it seems that the situation of mixed ideals is more involved; for example, for $\mathfrak{a}=\left(x^{2}-x y, y^{2}-x y, z^{2}-z w, w^{2}-z w\right)$, (taken from [loc. cit.]), we have $\operatorname{Ann}\left(H_{1}\right)=\overline{\mathfrak{a}}$, the integral closure, and $\operatorname{Ann}\left(H_{2}\right)=\sqrt{\mathfrak{a}}$. This example shows that the intersection of the annihilators has a better structure than the union. This is an interesting example for us since $(\mathfrak{a}: \overline{\mathfrak{a}})$ is a 4-residual intersection, with $\overline{\mathfrak{a}} / \mathfrak{a}$ cyclic and $\overline{\mathfrak{a}}$ satisfying SD.

Since a part of our results works well with arithmetic $s$-residual intersections, it is worth mentioning some general properties of these ideals.

Proposition 4.1. Let $R$ be a Noetherian ring, let $\mathfrak{a}=\left(a_{1}, \ldots, a_{s}\right) \subset I$ be an ideal of $R$ and let $J=\mathfrak{a}: I$ be an $s$-residual intersection. Then:

(i) $J$ is an arithmetic s-residual intersection if and only if $\operatorname{ht}\left(0: \bigwedge^{2}(I / \mathfrak{a})\right) \geq s+1$.

(ii) If 2 is unit in $R$, then $I+J \subseteq\left(0: \bigwedge^{2}(I / \mathfrak{a})\right)$.

(iii) The following statements are equivalent:

(1) For all $i \leq s$, an $i$-residual intersection of I exists.

(2) For any prime ideal $\mathfrak{p} \supset$ I such that $\mathrm{ht}(\mathfrak{p}) \leq s-1$, we have $\mu\left(I_{\mathfrak{p}}\right) \leq \mathrm{ht}(\mathfrak{p})+1$.

(3) There exist $a_{1}, \ldots, a_{s} \in I$ such that, for $i \leq s-1, J_{i}=\left(a_{1}, \ldots, a_{i}\right): I$ is an arithmetic $i$-residual intersection and $J=\left(a_{1}, \ldots, a_{s}\right): I$ is an (algebraic) s-residual intersection. 
(iv) $J$ is an arithmetic $s$-residual intersection if and only if there exists an element $b \in I$ such that $\mathrm{ht}((\mathfrak{a}, b): I) \geq s+1$.

Proof. For (i), $J=\mathfrak{a}: I$ is an arithmetic $s$-residual if and only if $\operatorname{ht}\left(\operatorname{Fitt}_{j}(I / \mathfrak{a})\right) \geq s+j$ for $j=0,1$ [Eisenbud 1995, Proposition 20.6]. Moreover, $\operatorname{Fitt}_{j}(I / \mathfrak{a})$ and the annihilator of $\bigwedge^{j+1}(I / \mathfrak{a})$ have the same radical [Eisenbud 1995, Exercise 20.10] which yields the assertion. Part (ii) is straightforward. Part (iii) goes along the same line as the proof of [Ulrich 1994, Lemma 1.4]. Part (iv) follows from [Ulrich 1994, Lemma 1.3].

The concept of $s$-parsimonious ideals first appeared in [Chardin et al. 2001], where it is shown in Proposition 3.1 that weakly $s$-residually $S_{2}$ ideals which satisfy $G_{s}$ are $s$-parsimonious. However, if $I$ is an ideal which satisfies the conditions of Corollary 2.8 then it follows from [Chardin et al. 2001, Proposition 3.3(a)] that $I$ is $s$-parsimonious.

Remark 4.2. Let $(R, \mathfrak{m})$ be a CM local ring of dimension $d$, and let $I$ be an ideal such that depth $(R / I) \geq d-s$ and such that $I$ satisfies any of the conditions in Theorem 2.6 for $k=1$. Then for any arithmetic $s$-residual intersection $J=(\mathfrak{a}: I)$, there exists an element $b \in I$ such that $J=(\mathfrak{a}: b)$.

This remark is further motivation to study the cases where $I / \mathfrak{a}$ is cyclic.

To exploit the structure of $H_{\bullet}\left({ }_{0} \mathcal{Z}_{\bullet}^{+}(\mathfrak{a} ; \mathfrak{a}, b)\right)$, the following lemma is crucial.

Lemma 4.3. Let $\mathfrak{a}=\left(a_{1}, \ldots, a_{s}\right), I=(\boldsymbol{f})=\left(b, a_{1}, \ldots, a_{s}\right)$, and consider the

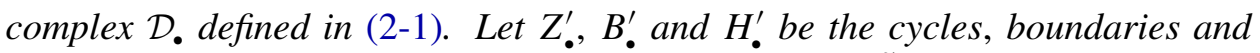
homologies of the Koszul complex $K_{\bullet}\left(a_{1}, \ldots, a_{s}\right)$. Put $\tilde{B}_{i}=\left(B_{i}^{\prime}:_{Z_{i}^{\prime}} b\right)$ and $S=$ $R\left[T_{0}, T_{1}, \ldots, T_{s}\right]$ with standard grading. Then:

(i) We have

$$
H_{i}\left(\mathcal{D}_{\bullet}\right) \cong \frac{\left(Z_{i}^{\prime} \otimes_{R} R\left[T_{0}\right]\right)(-i)}{\left(\tilde{B}_{i} \otimes_{R} T_{0} R\left[T_{0}\right]\right)(-i)}
$$

and, in particular,

$$
\left(H_{i}\left(\mathcal{D}_{\bullet}\right)_{T_{0}}\right)_{[0]} \cong b H_{i}\left(a_{1}, \ldots, a_{s}\right) \text { for all } i .
$$

(ii) If $R$ is a graded ring and $b, a_{1}, \ldots, a_{s}$ are homogeneous, then we have a homogeneous isomorphism

$$
\left(H_{i}\left(\mathcal{D}_{\bullet}\right)_{T_{0}}\right)_{[0]} \cong b H_{i}\left(a_{1}, \ldots, a_{s}\right)((i+1) \operatorname{deg}(b)) \text { for all } i .
$$

Proof. (i) To compute the homology modules of $\mathcal{D}_{\bullet}$, we consider the two spectral sequences arising from its double complex structure (2-1). We put this double complex in the second quadrant and locate $K_{0}(\boldsymbol{\gamma}) \otimes_{S} \mathcal{Z}_{0}(\boldsymbol{f})$ in the corner.

Set $S=R\left[T_{0}, T_{1}, \ldots, T_{S}\right]$, where $T_{0}$ corresponds to $b$ and $T_{i}$ to $a_{i}$. Then $\left(\gamma_{1} \cdots \gamma_{s}\right)=\left(T_{1} \cdots T_{s}\right)$ is a regular sequence on $\mathcal{Z}_{i}=Z_{i}\left[T_{0}, T_{1}, \ldots, T_{s}\right](-i)$, with 
$Z_{i}=Z_{i}\left(b, a_{1}, \ldots, a_{s}\right)$. We then have ${ }^{1} \mathrm{E}_{\mathrm{ver}}^{-i, j}=H_{j}\left(K .(\boldsymbol{\gamma}) \otimes \mathcal{Z}_{i}\right)=0$ for all $j \geq 1$ and all $i$. We also have $\left(S /\left(T_{1}, T_{2}, \ldots, T_{S}\right)\right) \otimes_{S} \mathcal{Z}_{i}=R\left[T_{0}\right] \otimes_{R} Z_{i}$, in which we consider $R\left[T_{0}\right]$ as an $S$-module by the trivial multiplication $T_{i} R\left[T_{0}\right]=0,1 \leq i \leq s$. It then follows that ${ }^{1} \mathrm{E}_{\mathrm{ver}}^{0, \bullet}$ is the complex

$0 \rightarrow Z_{s+1} \otimes_{R} R\left[T_{0}\right](-s-1) \stackrel{\partial_{s+1}}{\longrightarrow} \cdots \rightarrow Z_{1} \otimes_{R} R\left[T_{0}\right](-1)$

$$
\stackrel{\partial_{1}}{\longrightarrow} Z_{0} \otimes_{R} R\left[T_{0}\right] \rightarrow 0,
$$

wherein the differentials are induced by those in $\mathcal{Z} \cdot(f)$. As well,

$$
H_{i}\left({ }^{1} \mathrm{E}_{\mathrm{ver}}^{0, \bullet}\right)=H_{i}\left(\mathcal{Z} \bullet \otimes_{S}\left(S /\left(T_{1}, T_{2}, \ldots, T_{S}\right)\right)\right)(-i) .
$$

Considering the convergence of the spectral sequences to the homology module of the total complex $\mathcal{D}_{\bullet}$, we have $H_{i}\left(\mathcal{D}_{\bullet}\right)=H_{i}\left({ }^{1} \mathrm{E}_{\mathrm{ver}}^{0, \bullet}\right)=\operatorname{Ker}\left(\partial_{i}\right) / \operatorname{Im}\left(\partial_{i+1}\right)$. We compute this homology in two steps.

Step 1: $\left(\operatorname{Ker}\left(\partial_{i}\right)\right)$ Let $z_{i}=\left(\sum_{j} r_{j} e_{j_{1}} \wedge e_{j_{2}} \wedge \cdots \wedge e_{j_{i}}\right) \omega\left(T_{0}\right) \in Z_{i}\left[T_{0}\right](-i)$. We study two cases: first, if $j_{1} \neq 0$ for every $j$ which appears in the above presentation of $z_{i}$, then we have $z_{i} \in Z_{i}^{\prime}\left[T_{0}\right]$ and, moreover,

$$
\partial_{i}\left(z_{i}\right)=\sum_{j} r_{j}\left(\sum_{l=1}^{i} e_{j_{1}} \wedge \cdots \wedge e_{j_{l-1}} \wedge e_{j_{l+1}} \wedge \cdots \wedge e_{j_{i}} T_{l} \omega\left(T_{0}\right)\right)=0,
$$

where the last vanishing is due to the fact that $T_{i} R\left[T_{0}\right]=0$ for all $1 \leq i \leq s$. It follows that $z_{i} \in \operatorname{Ker}\left(\partial_{i}\right)$.

Second, we show that if $j_{1}=0$ for some $j$ that appears in $z_{i}$ then $z_{i} \notin \operatorname{Ker}\left(\partial_{i}\right)$. Writing $z_{i}=z_{i}^{\prime}+z_{i}^{\prime \prime} \wedge e_{0}$, where $z_{i}^{\prime}$ and $z_{i}^{\prime \prime}$ do not involve $e_{0}$, one has

$$
\partial_{i}\left(z_{i}\right)=\partial_{i}\left(z_{i}^{\prime}\right)+\partial_{i-1}\left(z_{i}^{\prime \prime}\right) \wedge e_{0}+(-1)^{i+1} T_{0} z_{i}^{\prime \prime}
$$

by the DG-algebra structure of $\left(\mathcal{Z}_{\bullet}, \partial_{\bullet}^{\prime}\right)$. Since $z_{i}^{\prime}$ and $z_{i}^{\prime \prime}$ do not involve $e_{0}$, it follows that $\partial_{i}\left(z_{i}^{\prime}\right)=0$ and $\partial_{i-1}\left(z_{i}^{\prime \prime}\right)=0$ by the multiplication on $R\left[T_{0}\right]$. Thus $\partial_{i}\left(z_{i}\right)=$ $(-1)^{i+1} T_{0} z_{i}^{\prime \prime} \neq 0$. Therefore, $\operatorname{ker}\left(\partial_{i}\right)=Z_{i}\left(a_{1}, \ldots, a_{s}\right)\left[T_{0}\right](-i)=Z_{i}^{\prime}\left[T_{0}\right](-i)$.

Step 2: $\left(\operatorname{Im}\left(\partial_{i+1}\right)\right)$ To compute the image, we consider an arbitrary element

$$
z_{i+1} \omega\left(T_{0}\right)=\left(\sum_{j} r_{j} e_{j_{1}} \wedge e_{j_{2}} \wedge \cdots \wedge e_{j_{i+1}}\right) \omega\left(T_{0}\right) \in Z_{i+1}\left[T_{0}\right](-i-1)
$$

and write $z_{i+1}=z_{i+1}^{\prime}+z_{i+1}^{\prime \prime} \wedge e_{0}$, where $z_{i+1}^{\prime}$ and $z_{i+1}^{\prime \prime}$ do not involve $e_{0}$. Recall that $Z_{i+1}=Z_{i+1}(f)$. Consider the Koszul complex $\left(K_{\bullet}(f), d_{\bullet}\right)$. We have

$$
0=d\left(z_{i+1}\right)=d\left(z_{i+1}^{\prime}\right)+d\left(z_{i+1}^{\prime \prime}\right) \wedge e_{0}+(-1)^{i} b z_{i+1}^{\prime \prime} .
$$

Since $z_{i+1}^{\prime}$ and $z_{i+1}^{\prime \prime}$ do not involve $e_{0}$, the above equation implies that $d\left(z_{i+1}^{\prime \prime}\right)=0$ and $b z_{i+1}^{\prime \prime}=d\left((-1)^{i+1} z_{i+1}^{\prime}\right)$, hence $z_{i+1}^{\prime \prime} \in\left(B_{i}^{\prime}: z_{i}^{\prime} b\right)$. 
Conversely, for any $z_{i+1}^{\prime \prime} \in\left(B_{i}^{\prime}: Z_{i}^{\prime} b\right)$, we have $b z_{i+1}^{\prime \prime}=d\left((-1)^{i+1} z_{i+1}^{\prime}\right)$ for some $z_{i+1}^{\prime} \in K_{i+1}^{\prime}$, hence $z_{i+1}=\left(z_{i+1}^{\prime}+z_{i+1}^{\prime \prime} \wedge e_{0}\right) \in Z_{i+1}$.

On the other hand, $\partial\left(z_{i+1}\right)=\partial\left(z_{i+1}^{\prime}\right)+\partial\left(z_{i+1}^{\prime \prime} \wedge e_{0}\right)=0+\partial\left(z_{i+1}^{\prime \prime} \wedge e_{0}\right)=$ $(-1)^{i} T_{0} z_{i+1}^{\prime \prime}$.

It follows that $\operatorname{Im}\left(\partial_{i+1}\right)=\left(T_{0} \tilde{B}_{i}\left[T_{0}\right]\right)(-i)$. Finally, the above two steps show that

$$
H_{i}\left(\mathcal{D}_{\bullet}\right)=\frac{\left(Z_{i}^{\prime} \otimes_{R} R\left[T_{0}\right]\right)(-i)}{\left(\tilde{B}_{i} \otimes_{R} T_{0} R\left[T_{0}\right]\right)(-i)} .
$$

To see the last assertion, just notice that

$$
\left(\left(Z_{i}^{\prime}\left[T_{0}\right]_{T_{0}}\right)(-i)\right)_{[0]}=Z_{i}^{\prime}\left[T_{0}, T_{0}^{-1}\right]_{[-i]}=T_{0}^{-i} Z_{i}^{\prime},
$$

and

$$
\left(\left(\tilde{B}_{i} T_{0}\left[T_{0}\right]_{T_{0}}\right)(-i)\right)_{[0]}=T_{0}^{-i} \tilde{B}_{i}
$$

Therefore,

$$
\left(H_{i}\left(\mathcal{D}_{\bullet}\right)_{T_{0}}\right)_{[0]} \simeq Z_{i}^{\prime} / \tilde{B}_{i}=Z_{i}^{\prime} /\left(B_{i}^{\prime}: Z_{i}^{\prime} b\right)=H_{i}^{\prime} /\left(0:_{H_{i}^{\prime}} b\right) \simeq b H_{i}\left(a_{1}, \ldots, a_{s}\right) .
$$

(ii) In the graded case, every homomorphism in the above argument is homogeneous except the first and last isomorphisms in (4-3). To see the desired shift, notice that the bidegree of $T_{0}$ is $(\operatorname{deg}(b), 1)$. Hence, considering $\left(H_{i}\left(\mathcal{D}_{\bullet}\right)_{T_{0}}\right)$ in bidegree $(0,0)$, we have

$$
\left(H_{i}\left(\mathcal{D}_{.}\right)_{T_{0}}\right)_{[(0,0)]} \simeq \frac{T_{0}^{-i}\left(\left(Z_{i}^{\prime}\right)_{[i \operatorname{deg}(b)]}\right)}{T_{0}^{-i}\left(\left(\tilde{B}_{i}\right)_{[i \operatorname{deg}(b)]}\right)} \simeq\left(\frac{Z_{i}^{\prime}}{\tilde{B}_{i}}\right)_{[i \operatorname{deg}(b)]} .
$$

Moreover, there exists the homogeneous exact sequence

$$
0 \rightarrow \tilde{B}_{i} \rightarrow Z_{i}^{\prime} \rightarrow b H_{i}\left(a_{1}, \ldots, a_{s}\right)(\operatorname{deg}(b)) \rightarrow 0,
$$

which yields the assertion.

Now, we are ready to explain our main theorem in this section. The dependence of the homology modules of ${ }_{0} \mathcal{Z}^{+}(\boldsymbol{a}, \boldsymbol{f})$ on the generating sets becomes clear by this theorem; furthermore, it motivates the study of the uniform annihilator of the Koszul homology modules.

Theorem 4.4. Let $R$ be $a$ (Noetherian) ring, and let $\mathfrak{a}=(\boldsymbol{a})=\left(a_{1}, \ldots, a_{s}\right)$ and $I=(\boldsymbol{f})=\left(b, a_{1}, \ldots, a_{s}\right)$. Then $H_{i}\left({ }_{0} \mathcal{Z}_{\bullet}^{+}(\boldsymbol{a}, \boldsymbol{f})\right) \simeq b H_{i}\left(a_{1}, \ldots, a_{s}\right)$ for all $i \geq 1$ and $H_{0}\left({ }_{0} \mathcal{Z}_{.}^{+}(\boldsymbol{a}, \boldsymbol{f})\right) \simeq R /(\mathfrak{a}: b)$; furthermore, in the graded case we have $H_{i}\left({ }_{0} \mathcal{Z}_{\bullet}^{+}(\boldsymbol{a}, \boldsymbol{f})\right) \simeq b H_{i}\left(a_{1}, \ldots, a_{s}\right)((i+1) \operatorname{deg}(b))$ for $i \geq 1$.

Proof. We study the spectral sequences arising from the third-quadrant double complex $C_{\mathfrak{g}}^{\bullet} \otimes \mathcal{D}$. with $C_{\mathfrak{g}}^{0} \otimes D_{0}$ in the center. Since $T_{0}, T_{1}, \ldots, T_{s}$ is a regular sequence on the $D_{i}$, we have $\left({ }^{1} \mathrm{E}_{\mathrm{ver}}\right)_{[0]}=H_{\mathfrak{g}}^{s+1}\left(\mathcal{D}_{\bullet}\right)_{[0]}$. The latter is $\mathcal{Z}_{\text {. }}^{+}$by definition (2-5), which is the truncated complex ${ }_{0} \mathcal{Z}_{\bullet}^{+}(\boldsymbol{a}, \boldsymbol{f})_{\geq 1}$. 
On the other hand, in the horizontal spectral sequence we deal with $H_{\mathfrak{g}}^{j}\left(H_{i}\left(\mathcal{D}_{\bullet}\right)\right)$. $H_{i}\left(\mathcal{D}_{\bullet}\right)$ is annihilated by $\left(T_{1}, \ldots, T_{S}\right)$ by $(4-2)$, which implies that $H_{\mathfrak{g}}^{j}\left(H_{i}\left(\mathcal{D}_{\bullet}\right)\right)=$ $H_{\left(T_{0}\right)}^{j}\left(H_{i}\left(\mathcal{D}_{\bullet}\right)\right)$ for all $i$ and $j$. Also notice that $H_{\left(T_{0}\right)}^{0}\left(H_{i}\left(\mathcal{D}_{\bullet}\right)\right)_{[0]}=0$ for $i \geq 1$, since $\operatorname{indeg}\left(D_{i}\right)=i$. Hence

$$
\left({ }^{2} \mathrm{E}_{\mathrm{hor}}^{-i,-j}\right)_{[0]}= \begin{cases}H_{\left(T_{0}\right)}^{0}\left(H_{0}\left(\mathcal{D}_{\bullet}\right)\right)_{[0]}, & i=0 \text { and } j=0, \\ H_{\left(T_{0}\right)}^{1}\left(H_{i}\left(\mathcal{D}_{\bullet}\right)\right)_{[0]}=\left(H_{i}\left(\mathcal{D}_{\bullet}\right)_{T_{0}}\right)_{[0]}, & i \geq 1 \text { and } j=1, \\ 0, & \text { otherwise. }\end{cases}
$$

Considering both spectrals at the same time, we have:

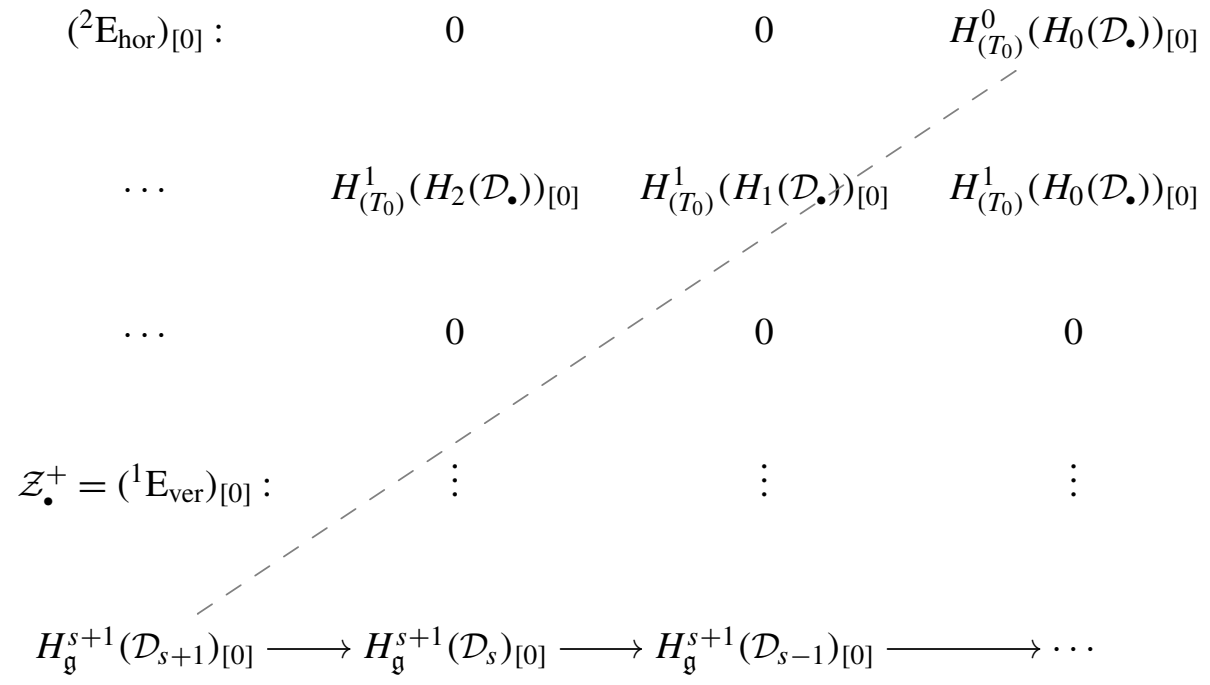

By the convergence of the spectral sequences, we have $H_{i+1}\left({ }_{0} \mathcal{Z}_{\bullet}^{+}\right)=H_{i}\left(\mathcal{Z}_{\bullet}^{+}\right)=$ $H_{\left(T_{0}\right)}^{1}\left(H_{i+1}\left(\mathcal{D}_{\bullet}\right)\right)_{[0]}$ for $i \geq 1$. Quite generally, $H_{\left(T_{0}\right)}^{1}\left(H_{i+1}\left(\mathcal{D}_{\bullet}\right)\right)_{[0]}$ is isomorphic to $\left(H_{i+1}\left(\mathcal{D}_{\bullet}\right)_{T_{0}}\right)_{[0]}$, which in turn is isomorphic to $b H_{i+1}\left(a_{1}, \ldots, a_{s}\right)$ by Lemma 4.3. In the case where $i=0$, the convergence of the spectral sequences provides the exact sequence

$$
0 \rightarrow H_{\left(T_{0}\right)}^{1}\left(H_{1}\left(\mathcal{D}_{\bullet}\right)\right)_{0} \rightarrow H_{0}\left(\mathcal{Z}_{\bullet}^{+}\right) \stackrel{\psi}{\longrightarrow} H_{\left(T_{0}\right)}^{0}\left(H_{0}\left(\mathcal{D}_{\bullet}\right)\right)_{0} \rightarrow 0 .
$$

According to (2-5), $H_{0}\left(\mathcal{Z}_{\bullet}^{+}\right)=\operatorname{Coker}\left(\phi_{0}\right)$, and $\psi$ is used to define $\tau_{0}$ in (2-7). Therefore, $\operatorname{Ker}(\psi)=H_{1}\left({ }_{0} \mathcal{Z}^{+}\right)$and its image determines $H_{0}\left({ }_{0} \mathcal{Z}_{\bullet}^{+}\right)$, which we will compute below.

Since the horizontal spectral stabilizes in the second step, we have ${ }^{\infty} \mathrm{E}_{\text {hor }}^{0,0}=$ ${ }^{2} \mathrm{E}_{\text {hor }}^{0,0}=H_{\left(T_{0}\right)}^{0}\left(H_{0}\left(\mathcal{D}_{\bullet}\right)\right)$. Moreover, end $\left(H_{\mathfrak{g}}^{s+1}\left(\mathcal{D}_{s+1}\right)\right)=0$, thus $\left({ }^{\infty} \mathrm{E}_{\text {hor }}^{0,0}\right)_{[i]}=0$ for $i \geq 1$, which in particular implies that $H_{\left(T_{0}\right)}^{0}\left(H_{0}\left(\mathcal{D}_{\bullet}\right)\right)_{[1]}=0$. Specifically, we then have $T_{0} H_{\left(T_{0}\right)}^{0}\left(H_{0}\left(\mathcal{D}_{\bullet}\right)\right)_{[0]}=0$. Recall that $H_{0}\left(\mathcal{D}_{\bullet}\right)=\operatorname{Sym}(I) / \boldsymbol{\gamma} \operatorname{Sym}(I)$ and that we consider $\mathcal{S}_{I}:=\operatorname{Sym}_{R}(I)$ as an $S=R\left[T_{0}, \ldots, T_{S}\right]$-module via the ring 
homomorphism $S \rightarrow \mathcal{S}_{I}$ sending $T_{0}$ to $b$ and $T_{i}$ to $a_{i}$ as an element of $\left(\mathcal{S}_{I}\right)_{[1]}=I$. Hence $T_{0} H_{\left(T_{0}\right)}^{0}\left(H_{0}\left(\mathcal{D}_{\bullet}\right)\right)_{[0]}=0$ as an element in $(\operatorname{Sym}(I) / \boldsymbol{\gamma} \operatorname{Sym}(I))_{[1]}=I / \mathfrak{a}$ is equivalent to saying that $H_{\left(T_{0}\right)}^{0}\left(H_{0}\left(\mathcal{D}_{\bullet}\right)\right)_{[0]} \subseteq(\mathfrak{a}: b)$.

On the other hand, $H_{\left(T_{0}\right)}^{0}\left(H_{0}\left(\mathcal{D}_{\bullet}\right)\right)_{[0]}=(\mathfrak{a}: b) \cup\left(\bigcup_{i=1}^{\infty}\left(\mathfrak{a} \operatorname{Sym}_{R}^{i}(I):_{R} \operatorname{Sym}_{R}^{i+1}(I)\right)\right)$, hence $H_{\left(T_{0}\right)}^{0}\left(H_{0}\left(\mathcal{D}_{\bullet}\right)\right)_{0} \supseteq(\mathfrak{a}: b)$, which yields $H_{\left(T_{0}\right)}^{0}\left(H_{0}\left(\mathcal{D}_{\bullet}\right)\right)_{[0]}=(\mathfrak{a}: b)$.

Further, by Lemma $4.3, H_{\left(T_{0}\right)}^{1}\left(H_{1}\left(\mathcal{D}_{\bullet}\right)\right)_{[0]}=\left(H_{1}\left(\mathcal{D}_{\bullet}\right)_{T_{0}}\right)_{[0]}=b H_{1}\left(f_{1}, f_{2}, \ldots, f_{s}\right)$. Substituting these facts in the short exact sequence (4-5), the assertion follows.

As a summary we have the following corollary.

Corollary 4.5. Let $R$ be a (Noetherian) ring, and let $\mathfrak{a}=(\boldsymbol{a})=\left(a_{1}, \ldots, a_{s}\right)$ and $I=(\boldsymbol{f})=\left(b, a_{1}, \ldots, a_{s}\right)$. If $b H_{i}\left(a_{1}, \ldots, a_{s}\right)=0$ for all $i \geq 1$, then there exists an exact complex

$$
0 \rightarrow \mathcal{Z}_{s}^{+} \rightarrow \mathcal{Z}_{s-1}^{+} \rightarrow \cdots \rightarrow \mathcal{Z}_{0}^{+} \rightarrow\left(\mathfrak{a}:_{R} b\right) \rightarrow 0,
$$

wherein $\mathcal{Z}_{i}^{+}=\bigoplus_{j=i+1}^{r} Z_{j}(f)^{\oplus n_{j}}$ for some positive integers $n_{j}$ and $r$. In particular, the index of the lowest cycle which appears in the components increases along the complex.

Theorem 4.4, in conjunction with other conditions which imply the acyclicity of the complex ${ }_{0} \mathcal{Z}_{\bullet}^{+}$, yields nontrivial facts about the uniform annihilators of nonzero Koszul homologies.

Corollary 4.6. Let $(R, \mathfrak{m})$ be a $C M$ local ring of dimension $d$, and suppose that $I=\left(b, a_{1}, \ldots, a_{s}\right)$ satisfies $S D$. If $\operatorname{dim}\left(b H_{i}\left(a_{1}, \ldots, a_{s}\right)\right) \leq d-s$ for all $i \geq 1$ then

- $b H_{i}\left(a_{1}, \ldots, a_{s}\right)=0$ for all $i \geq 1$, and

- $\operatorname{depth}\left(R /\left(\left(a_{1}, \ldots, a_{s}\right): I\right)\right) \geq d-s$.

Proof. We consider the complex ${ }_{0} \mathcal{Z}_{.}^{+}(\mathfrak{a} ;(b, \mathfrak{a}))$. By Theorem 4.4, the hypothesis on the dimensions implies that this complex is acyclic locally at codimension $s-1$. Then, appealing to the acyclicity lemma, the sliding depth hypothesis shows that the complex is acyclic on the punctured spectrum and, finally, acyclic (the same technique which we used to prove Proposition 2.5). Once more applying Theorem 4.4, we conclude the first assertion. The depth inequality follows from the proof of Theorem 2.6.

As a final remark in this section, we notice that if $(\mathfrak{a}: I)$ is an $s$-residual intersection then $\operatorname{dim}\left(I H_{i}(\mathfrak{a})\right) \leq d-s$ for all $i$. Thus, the latter property may be considered as a generalization of algebraic residual intersection.

\section{Uniform annihilator of Koszul homologies}

Motivated by the results in the previous section, we concentrate on the uniform annihilator of Koszul homologies in this section. In the main theorem of this 
section, we see that in any residual intersection $J=\mathfrak{a}: I$ the sliding depth condition passes from $I$ to $\mathfrak{a}$. This fact was known to experts only in the presence of the $G_{\infty}$ condition [Ulrich 1994, 1.8(3)+1.12], which is not that surprising since $I$ is then generated by a $d$-sequence. However, it was unknown even for perfect ideals of height 2 which are not $G_{\infty}$; see [Eisenbud and Ulrich 2016] for such an example.

The next interesting result in this section is Corollary 5.6, in which we show that, for any residual intersection $J=\mathfrak{a}: I$ with $I$ satisfying sliding depth, $I$ is contained in the uniform annihilator of the nonzero Koszul homologies of $\mathfrak{a}$. Hence this is a kind of universal property for such ideals.

The next proposition is fundamental to proving the aforementioned results, though it is also interesting by itself.

Proposition 5.1. Let $(R, \mathfrak{m})$ be a CM local ring of dimension $d$, and let $\mathfrak{a}=$ $\left(f_{1}, f_{2}, \ldots, f_{s}\right)$ and $I=\left(f_{1}, f_{2}, \ldots, f_{r}\right)$ be ideals with $s \leq r$. Let $J=(\mathfrak{a}: I)$ and $g=\mathrm{ht}(I)=\mathrm{ht}(\mathfrak{a}) \geq 1$. Let $n$ be an integer and suppose that $\mathrm{ht}(J) \geq n \geq 1$ and that depth $\left(H_{l}\left(f_{1}, f_{2}, \ldots, f_{r}\right)\right) \geq d-r+j$ for $l \geq r-n+2$. Then, for any $k$ with $r \geq k \geq s$ and any $j \geq k-n+2$ :

(i) $\operatorname{depth}\left(H_{j}\left(f_{1}, f_{2}, \ldots, f_{k}\right) \geq d-k+j\right.$, and

(ii) $I \subseteq \operatorname{Ann}\left(H_{j}\left(f_{1}, f_{2}, \ldots, f_{k}\right)\right)$.

Both assertions hold for sequences of the form $\left(f_{1}, \ldots, f_{s}, f_{i_{1}}, \ldots, f_{i_{k-s}}\right)$, where $\left\{f_{i_{1}}, \ldots, f_{i_{k-s}}\right\} \subseteq\left\{f_{s+1}, \ldots, f_{r}\right\}$.

Proof. The proof is by a recursive induction on $k$. By assumption, the result is true for $k=r$. Suppose that it is true for $k \leq r$. We now apply a recursive induction on $j \geq(k-1)-n+2$ to show the following claims:

(1) $f_{k} H_{j}\left(f_{1}, f_{2}, \ldots, f_{k-1}\right)=0$;

(2) $\operatorname{depth}\left(H_{j}\left(f_{1}, f_{2}, \ldots, f_{k-1}\right)\right) \geq d-(k-1)+j$;

(3) $\operatorname{depth}\left(Z_{j}\left(f_{1}, f_{2}, \ldots, f_{k-1}\right)\right) \geq d-(k-1)+j$;

(4) $\operatorname{depth}\left(B_{j}\left(f_{1}, f_{2}, \ldots, f_{k-1}\right)\right) \geq d-(k-1)+j$.

Since these claims are clear for $j>k-g$, we consider an integer $j \geq(k-1)-n+3$ and we suppose, by induction hypothesis, that the result holds for $j$. A simple depth-chasing between cycles, boundaries and homologies shows that (2)-(4) for $j-1$ follow from (1) for $j-1$ and the induction hypotheses of (2)-(4) for $j$. We just notice that condition (1) provides the exact sequence

$$
0 \rightarrow H_{j}\left(f_{1}, \ldots, f_{k-1}\right) \rightarrow H_{j}\left(f_{1}, \ldots, f_{k}\right) \rightarrow H_{j-1}\left(f_{1}, \ldots, f_{k-1}\right) \rightarrow 0 .
$$

We then proceed to prove (1) for $j-1$ while assuming the four claims hold for $j$. To do this, we show that $f_{k} H_{j-1}\left(f_{1}, f_{2}, \ldots, f_{k-1}\right)_{\mathfrak{p}}=0$ by an induction on $\operatorname{ht}(\mathfrak{p})$. For ht $(\mathfrak{p})<n$ it is clear, since $I_{\mathfrak{p}}=a_{\mathfrak{p}}$. Now let $\mathfrak{q}$ be a prime ideal with ht(q) $\geq n$ 
and suppose that (1)-(4) hold locally for all $\mathfrak{p}$ such that $\mathfrak{p} \subset \mathfrak{q}$. We may, and will, replace $(R, \mathfrak{m})$ by $\left(R_{\mathfrak{q}}, \mathfrak{q} R_{\mathfrak{q}}\right)$. Considering the exact sequence

$$
0 \rightarrow Z_{j}\left(f_{1}, \ldots, f_{k-1}\right) \rightarrow \bigwedge^{j}\left(R^{k-1}\right) \rightarrow B_{j-1}\left(f_{1}, \ldots, f_{k-1}\right) \rightarrow 0,
$$

out of the Koszul complex, we obtain

$$
\operatorname{depth}\left(B_{j-1}\right) \geq \min \left\{\operatorname{depth}\left(\bigwedge^{j}\left(R^{k-1}\right)\right) \operatorname{depth}\left(Z_{j}\right)-1\right\} \geq d-(k-1)+j-1 .
$$

By assumption, $j \geq k-n+2$, which implies that $\operatorname{depth}\left(B_{j-1}\left(f_{1}, \ldots, f_{k-1}\right)\right) \geq$ $d-n+2 \geq 2$. This time,

$$
0 \rightarrow B_{j-1}\left(f_{1}, \ldots, f_{k-1}\right) \rightarrow Z_{j-1}\left(f_{1}, \ldots, f_{k-1}\right) \rightarrow H_{j-1}\left(f_{1}, \ldots, f_{k-1}\right) \rightarrow 0
$$

implies that $\operatorname{depth}\left(H_{j-1}\right) \geq 1, \operatorname{since} \operatorname{depth}\left(Z_{j-1}\right) \geq 1, \operatorname{as} \operatorname{dim}\left(R_{\mathfrak{q}}\right) \geq n \geq 1$.

We now consider the exact sequence

$$
0 \rightarrow H_{j}\left(f_{1}, \ldots, f_{k-1}\right) \rightarrow H_{j}\left(f_{1}, \ldots, f_{k-1}, f_{k}\right) \rightarrow \Gamma_{j-1} \rightarrow 0,
$$

where $\Gamma_{j-1}=\left(0:_{H_{j-1}}\left(f_{1}, \ldots, f_{k-1}\right) f_{k}\right)$.

The induction hypotheses imply that

$$
\begin{aligned}
\operatorname{depth}\left(\Gamma_{j-1}\right) & \geq \min \left\{\operatorname{depth}\left(H_{j}\left(f_{1}, \ldots, f_{k-1}, f_{k}\right)\right), \operatorname{depth}\left(H_{j}\left(f_{1}, \ldots, f_{k-1}\right)\right)-1\right\} \\
& \geq \min \{d-k+j, d-(k-1)+j-1\} \geq d-k+j \\
& \geq d-k+(k-n+2)=d-n+2 \geq 2 .
\end{aligned}
$$

At last, we have the inclusion $\Gamma_{j-1} \subseteq H_{j-1}$, where the former has depth at least 2 and the latter has depth at least 1, while the equality holds on the punctured spectrum, by the induction hypothesis. Therefore, this inclusion must be an equality, which proves claim (1) for $j-1$.

Applying the above argument for $k=r$, we have $\operatorname{depth}\left(H_{j}\left(f_{1}, \ldots, f_{r-1}\right)\right) \geq$ $d-(r-1)+j$ for all $j \geq(r-1)-n+2$, and $f_{r} H_{j}\left(f_{1}, f_{2}, \ldots, f_{r-1}\right)=0$. However, as long as we fix $f_{1}, \ldots, f_{s}$ we may change the role of $f_{r}$ with any one of $\left\{f_{s+1}, \ldots, f_{r}\right\}$. In other words, we have $\operatorname{depth}\left(H_{j}\left(f_{1}, \ldots, f_{s}, \ldots, \hat{f}_{i}, \ldots, f_{r}\right)\right) \geq$ $d-(r-1)+j$ for all $j \geq(r-1)-n+2$, and $f_{i} H_{j}\left(f_{1}, \ldots, f_{s}, \ldots, \hat{f}_{i}, \ldots, f_{r}\right)=0$. notice that, for any ideal $\mathfrak{a} \subseteq I^{\prime} \subseteq I$, we have ht $\left(\mathfrak{a}: I^{\prime}\right) \geq \operatorname{ht}(\mathfrak{a}: I) \geq n$. Therefore, if (i) holds for $\left(f_{1}, \ldots, f_{s}, f_{i_{1}}, \ldots, f_{i_{k-s}}\right)$, where $\left\{f_{i_{1}}, \ldots, f_{i_{k-s}}\right\} \subseteq\left\{f_{s+1}, \ldots, f_{r}\right\}$, then the above argument shows that (i) holds for $\left(f_{1}, \ldots, f_{s}, f_{i_{1}}, \ldots, f_{i_{k-s-1}}\right)$ and, moreover, that $f_{i_{k-s}} H_{j}\left(f_{1}, \ldots, f_{s}, f_{i_{1}}, \ldots, f_{i_{k-s-1}}\right)=0$ for $j \geq k-1-n+2$. Since $f_{i_{k-s}}$ varies over the set $\left\{f_{s+1}, \ldots, f_{r}\right\}$, we must have $I H_{j}\left(f_{1}, \ldots, f_{s}, f_{i_{1}}, \ldots, f_{i_{k-s-1}}\right)=0$ for $j \geq k-1-n+2$, which completes the proof.

We next want to show, in Lemma 5.3, that under the sliding depth condition there exists a similar depth inequality for cycles of the Koszul complex. For this, 
we need the following lemma which is reminiscent of the inductive Koszul long exact sequence.

Lemma 5.2. Let $R$ be a commutative ring, let $\left\{f_{0}, f_{1}, \ldots, f_{r}\right\} \subseteq R, Z$. B. be the Koszul cycles and boundaries with respect to the sequence $\left\{f_{1}, f_{2}, \ldots, f_{r}\right\}$, let $Z_{\text {. }}^{\prime}$ be the Koszul cycles with respect to the sequence $\left(f_{0}, f_{1}, \ldots, f_{r}\right)$ and let $\Gamma_{\bullet}=\left(B_{\bullet}:_{\bullet} f_{0}\right)$. Then, for any $j \geq 0$, there exists an exact sequence

$$
0 \rightarrow Z_{j} \rightarrow Z_{j}^{\prime} \rightarrow \Gamma_{j-1} \rightarrow 0 .
$$

Proof. The Koszul complex $K_{\bullet}\left(f_{1}, f_{2}, \ldots, f_{r}\right)$ is canonically a subcomplex of $K .\left(f_{0}, f_{1}, \ldots, f_{r}\right)$. We will denote the differential of both complexes by $d$. Let $\left\{e_{0}, e_{1}, \ldots, e_{r}\right\}$ be the canonical basis of $R^{r+1}$. Every $\theta \in Z_{j}^{\prime}$ can be written uniquely as a $\operatorname{sum} \theta=e_{0} \wedge w+v$, where $e_{0}$ does not appear in terms $w$ and $v$. Since $\theta \in Z_{j}^{\prime}$,

$$
0=d(\theta)=d\left(e_{0} \wedge w+v\right)=f_{0} w-e_{0} \wedge d(w)+d(v) .
$$

It follows that

$$
\left\{\begin{array} { l } 
{ e _ { 0 } \wedge d ( w ) = 0 } \\
{ f _ { 0 } w = - d ( v ) }
\end{array} \text { hence } \left\{\begin{array}{l}
d(w)=0 \\
f_{0} w=-d(v)
\end{array} \text { therefore } \quad w \in \Gamma_{j-1} .\right.\right.
$$

We define $\varphi: Z_{j}^{\prime} \rightarrow \Gamma_{j-1}$ by $\varphi(\theta)=\varphi\left(e_{0} \wedge w+v\right)=w$. The homomorphism $\varphi$ is well-defined, as the expression is unique. It is onto, since for any $w \in \Gamma_{j-1}$ there is a $v \in K_{j}\left(f_{1}, f_{2}, \ldots, f_{r}\right)$ such that $f_{0} w=-d(v)$. Hence $\theta=e_{0} \wedge w+v \in$ $K_{j}\left(f_{0}, f_{1}, \ldots, f_{r}\right)$ is the preimage of $w$.

Moreover, $Z_{j}=\operatorname{ker}(\varphi)$. On one hand, if $\theta \in Z_{j}^{\prime}$ belongs to $Z_{j}$, then there is no $e_{0}$ in its decomposition in terms of the canonical basis of $R^{r+1}$. Consequently, $\theta=e_{0} \wedge w+v=v$ and $w=0$. On the other hand, if $0=d(\theta)=d\left(e_{0} \wedge w+v\right)=w$, then $\theta=v$. Therefore, $\theta \in Z_{j}^{\prime} \cap K_{j}\left(f_{1}, f_{2}, \ldots, f_{r}\right)=Z_{j}$.

The next lemma is needed in the proof of Theorem 5.4. Notice that the sliding depth condition does not pass automatically from homologies to cycles because there is a level restriction; see Proposition 2.4.

Lemma 5.3. Let $(R, \mathfrak{m})$ be a CM local ring of dimension d. Let $\mathfrak{a}=\left(f_{1}, f_{2}, \ldots, f_{s}\right)$ and $I=\left(f_{1}, f_{2}, \ldots, f_{r}\right)$ be ideals with $s \leq r$, and let $J=(\mathfrak{a}: I)$ and $g=\mathrm{ht}(I)=$ $\mathrm{ht}(\mathfrak{a}) \geq 1$. Suppose that $h t(J) \geq n \geq 1$ and that $I$ satisfies $\mathrm{SD}$. Then, for $k \geq s$ and $j \geq k-n+2$,

$$
\operatorname{depth}\left(Z_{j}\left(f_{1}, f_{2}, \ldots, f_{k}\right)\right) \geq d-k+j+1 .
$$

Proof. By Proposition 5.1, $I H_{j}\left(f_{1}, f_{2}, \ldots, f_{k}\right)=0$ whenever $s \leq k \leq r$ and $j \geq k-n+2$. Therefore, by Lemma 5.2, we have the following exact sequence for $k-1 \geq s$ and $j-1 \geq(k-1)-n+2$ :

$0 \rightarrow Z_{j}\left(f_{1}, f_{2}, \ldots, f_{k-1}\right) \rightarrow Z_{j}\left(f_{1}, f_{2}, \ldots, f_{k}\right) \rightarrow Z_{j-1}\left(f_{1}, f_{2}, \ldots, f_{k-1}\right) \rightarrow 0$. 
We will prove the result by a recursive induction on $k$. If $k=r$, then it follows from Proposition 2.4. Suppose that the result holds for $k \geq s+1$. Since $Z_{j}\left(f_{1}, f_{2}, \ldots, f_{k-1}\right)$ satisfies $\mathrm{SDC}_{1}$ for $j \geq(k-1)-g+1$, we can use a new induction on $j$ to conclude that

$$
\operatorname{depth}\left(Z_{j-1}\left(f_{1}, f_{2}, \ldots, f_{k-1}\right)\right) \geq d-(k-1)+(j-1)+1=d-k+j+1 .
$$

We are now ready to prove the main theorem of this section. As we mentioned at the beginning of the section, this fact was known only in the presence of the $G_{\infty}$ condition, under which the problem reduced to studying the properties of ideals generated by $d$-sequences. Here the only assumption is the sliding depth and that $\operatorname{depth}(R / I) \geq d-s$.

Theorem 5.4. Let $(R, \mathfrak{m})$ be a $C M$ local ring of dimension d and let $J=(\mathfrak{a}: I)$ be an $s$-residual intersection with $s \geq \mathrm{ht}(I) \geq 1$. If I satisfies $\mathrm{SD}$ and $\operatorname{depth}(R / I) \geq d-s$, then $\mathfrak{a}$ satisfies $\mathrm{SD}$ as well.

Proof. We apply Lemma 5.3 for $n=s$. It then follows that

$$
\operatorname{depth}\left(Z_{j}\left(f_{1}, f_{2}, \ldots, f_{s}\right)\right) \geq d-s+j+1
$$

for $j \geq 2$. The hypothesis $\operatorname{depth}(R / I) \geq d-s$ enables us to apply Corollary 2.8, which in turn implies that $\operatorname{depth}(R / \mathfrak{a}) \geq d-s$. Therefore, $\operatorname{depth}(\mathfrak{a}) \geq d-s+1$ and hence $\operatorname{depth}\left(Z_{1}(\mathfrak{a})\right) \geq d-s+2$; that is, $\mathfrak{a}$ satisfies $\mathrm{SDC}_{1}$, which is equivalent to SD by Proposition 2.4.

In the next proposition we prove a partial converse of Theorem 5.4.

Proposition 5.5. Let $(R, \mathfrak{m})$ be a $C M$ local ring of dimension $d$, and let $\mathfrak{a}=$ $\left(f_{1}, f_{2}, \ldots, f_{s}\right)$ and $I=\left(f_{1}, f_{2}, \ldots, f_{r}\right)$ with $s \leq r$. Let $J=(\mathfrak{a}: I)$ be an s-residual intersection. If $\mathfrak{a}$ satisfies $\mathrm{SD}$, then, for any $s \leq k \leq r$ and $j \geq k-s+1$ :

(i) $\operatorname{depth}\left(H_{j}\left(f_{1}, f_{2}, \ldots, f_{k}\right)\right) \geq d-k+j$, and

(ii) $\operatorname{depth}\left(Z_{j}\left(f_{1}, f_{2}, \ldots, f_{k}\right)\right) \geq d-k+j+1$.

Proof. We will use induction on $k$. If $k=s$, then (ii) is clear by Proposition 2.4. Suppose that the result holds for $k \geq s+1$. Then, by the induction hypothesis, we have

$$
\operatorname{depth}\left(H_{j}\left(f_{1}, f_{2}, \ldots, f_{k}\right)\right) \geq d-k+j \geq d-s+1 .
$$

By a similar argument as in the proof of Proposition $5.1, I H_{j}\left(f_{1}, f_{2}, \ldots, f_{k}\right)=0$ for all $j \geq k-s+1$. Then we have the short exact sequences

$$
0 \rightarrow H_{j}\left(f_{1}, f_{2}, \ldots, f_{k}\right) \rightarrow H_{j}\left(f_{1}, f_{2}, \ldots, f_{k+1}\right) \rightarrow H_{j-1}\left(f_{1}, f_{2}, \ldots, f_{k}\right) \rightarrow 0
$$

and

$$
0 \rightarrow Z_{j}\left(f_{1}, f_{2}, \ldots, f_{k}\right) \rightarrow Z_{j}\left(f_{1}, f_{2}, \ldots, f_{k+1}\right) \rightarrow Z_{j-1}\left(f_{1}, f_{2}, \ldots, f_{k}\right) \rightarrow 0,
$$


provided by Lemma 5.2 for any $j \geq k+1-s+1$.

A depth-chasing then completes the proof.

The next corollary of Theorem 5.4 shows a tight relation between the uniform annihilator of nonzero Koszul homology modules and residual intersections.

Corollary 5.6. Let $(R, \mathfrak{m})$ be a $C M$ local ring of dimension $d$, and let I satisfy $\mathrm{SD}$ and $\operatorname{depth}(R / I) \geq d-s$ with $s \geq \operatorname{ht}(I) \geq 1$. Let $J=(\mathfrak{a}: I)$ be an $s$-residual intersection and use $H_{j}(\mathfrak{a})$ to denote the $j$-th Koszul homology module with respect to a minimal generating set of $\mathfrak{a}$. Then

$$
I \subseteq \bigcap_{j \geq 1} \operatorname{Ann}\left(H_{j}(\mathfrak{a})\right) .
$$

Furthermore, the equality happens in the following cases, if $\operatorname{depth}(R / I) \geq d-s+1$ (hence $s \geq g+1$ ):

- If $\mu\left(I_{\mathfrak{p}}\right) \leq s-i$ for a fixed positive integer $i$ and for all $\mathfrak{p} \in \operatorname{Ass}(R / I)$, then $I=\operatorname{Ann}\left(H_{j}(\mathfrak{a})\right)$ for all $1 \leq j \leq i$.

- If $R$ is Gorenstein and $\operatorname{Ass}(R / I)=\min (R / I)$, then $I=\bigcap_{j \geq 1} \operatorname{Ann}\left(H_{j}(\mathfrak{a})\right)$.

Proof. By Corollary 2.8, $\mathfrak{a}$ is minimally generated by $s$ elements. According to Theorem 5.4, for any $j$, we have $\operatorname{depth}\left(H_{j}(\mathfrak{a})\right) \geq d-s+j$. In particular, ht $(\mathfrak{p}) \leq s-1$ for any $\mathfrak{p} \in \operatorname{Ass}\left(H_{j}(\mathfrak{a})\right)$ with $j \geq 1$; see [Bruns and Herzog 1998, 1.2.13].

To show that $I H_{j}(\mathfrak{a})=0$, we show that it has no associated prime. Notice that $I H_{j}(\mathfrak{a}) \subseteq H_{j}(\mathfrak{a})$, hence, for any $\mathfrak{p} \in \operatorname{Ass}\left(I H_{j}(\mathfrak{a})\right)$, we have $h t(\mathfrak{p}) \leq s-1$ for which $I_{\mathfrak{p}}=\mathfrak{a}_{\mathfrak{p}}$. Therefore, $\left(I H_{j}(\mathfrak{a})\right)_{\mathfrak{p}}=0$ as desired.

To see the equality, we localize at associated primes of $I$ which have height at most $s-1$, so that $I_{\mathfrak{p}}=\mathfrak{a}_{\mathfrak{p}}$ for them. Now let $\left(a_{1}, \ldots, a_{s}\right)$ be a minimal generating set of $\mathfrak{a}$. If $\mu\left(I_{\mathfrak{p}}\right) \leq s-i$ then $H_{j}\left(a_{1}, \ldots, a_{s}\right)_{\mathfrak{p}}$ has a direct summand of $(R / \mathfrak{a})_{\mathfrak{p}}$ for all $1 \leq j \leq i$, and thus its annihilator is $\mathfrak{a}_{\mathfrak{p}}=I_{\mathfrak{p}}$. In the case where $R$ is Gorenstein and $\operatorname{Ass}(R / I)=\min (R / I)$, we see that, locally at the associated primes of $R / I$, $I_{\mathfrak{p}}$ is unmixed and, moreover, $\left(a_{1}, \ldots, a_{s}\right)_{\mathfrak{p}}$ is not a regular sequence since $s>\operatorname{ht}(\mathfrak{p})$. Hence, for $t=\operatorname{ht}\left(I_{\mathfrak{p}}\right), \operatorname{Ann}\left(H_{s-t}\left(\mathfrak{a}_{\mathfrak{p}}\right)\right)$ is the unmixed part of $\mathfrak{a}_{\mathfrak{p}}=I_{\mathfrak{p}}$ which is $I_{\mathfrak{p}}$.

The next amazing example shows a benefit of relaxing the $G_{s}$ condition in our theorems. However, we took this example from [Eisenbud and Ulrich 2016] wherein the authors used it to show the necessity of the $G_{s}$ condition in their theorems.

Example 5.7. Let $(R, \mathfrak{m})=\mathbb{Q}\left[x_{1}, \ldots, x_{5}\right]_{\left(x_{1}, \ldots, x_{5}\right)}$ and let $I=I_{4}(N)$, where

$$
N=\left(\begin{array}{cccc}
x_{2} & x_{3} & x_{4} & x_{5} \\
x_{1} & x_{2} & x_{3} & x_{4} \\
0 & x_{1} & x_{2} & 0 \\
0 & 0 & x_{1} & x_{2} \\
0 & 0 & 0 & x_{1}
\end{array}\right)
$$


Let $\Delta_{i}$ be the minors obtained by omitting the $i$-th row of $N$. We then consider $\mathfrak{a}$ to be the ideal generated by entries of

$$
\left(\begin{array}{llll}
\Delta_{1}-\Delta_{2} & \Delta_{3} & -\Delta_{4} & \Delta_{5}
\end{array}\right)\left(\begin{array}{ccccc}
x_{1} & 0 & 0 & 0 & 0 \\
0 & x_{5} & 0 & 0 & 0 \\
x_{3} & x_{4} & x_{5} & x_{3} & 0 \\
0 & x_{3} & x_{4} & x_{5} & 0 \\
0 & x_{2} & x_{3} & x_{4} & x_{5}
\end{array}\right) .
$$

$J=(\mathfrak{a}: I)$ is a 5-residual intersection of $I$, and $I$ is a perfect ideal of height 2 hence it is SCM. Easily, one can see that any prime that contains $I$ must contain $\mathfrak{p}=\left(x_{1}, x_{2}\right)$; that is, $I$ is $\mathfrak{p}$-primary, since it is unmixed. The minimal presentation of $I$ is given by $N$. Therefore, $\operatorname{Fitt}_{4}(I)=\mathfrak{m}$, and $\operatorname{Fitt}_{3}(I) \not \subset \mathfrak{p}$ because $x_{4}^{2}-x_{3} x_{5} \notin \mathfrak{p}$, and $\operatorname{Fitt}_{2}(I) \subseteq \mathfrak{p}$ because any combination of 3 "diagonal" elements contains either 0 or $x_{1}$ or $x_{2}$. Thus $\mu\left(I_{\mathfrak{p}}\right)=3$; in particular, $I$ does not satisfy $G_{3}$. However, it completely suits our Corollary 5.6 for $s=5$ and $i=2$, hence $\operatorname{Ann}\left(H_{1}(\mathfrak{a})\right)=\operatorname{Ann}\left(H_{2}(\mathfrak{a})\right)=I$. Also, since ht $(J)=5$, any prime ideal of height 2 containing $\mathfrak{a}$ must contain $I$, which means $I=\mathfrak{a}^{\text {unm }}$. Since the ring is Gorenstein, we then have $I=\mathfrak{a}^{\text {unm }}=\operatorname{Ann}\left(H_{3}(\mathfrak{a})\right)$ (our Macaulay2 system was not able to calculate $\operatorname{Ann}\left(H_{1}(\mathfrak{a})\right)$ directly!).

We notice that Corollary 5.6 presents a universal property for any ideal $\mathfrak{a}$ such that $J=(\mathfrak{a}: I)$ is an $s$-residual intersection of $I$. For instance, in the above example, for any other $\mathfrak{a}$ such that $J=(\mathfrak{a}: I)$ is a 5-residual intersection of $I$, we have $\operatorname{Ann}\left(H_{1}(\mathfrak{a})\right)=\operatorname{Ann}\left(H_{2}(\mathfrak{a})\right)=\operatorname{Ann}\left(H_{3}(\mathfrak{a})\right)=\mathfrak{a}^{u n m}=I$.

An unpublished result of G.Levin [Vasconcelos 2005, Theorem 5.26] yields in [Corso et al. 2006, Corollary 2.7] that if $R$ is a Noetherian ring and $\mathfrak{a}$ is an ideal of finite projective dimension $n$, then $\left(\operatorname{Ann}\left(H_{1}(\mathfrak{a})\right)\right)^{n+1} \subseteq \mathfrak{a}$. Combining this result with Corollary 5.6, one faces the strange fact that $\mathfrak{a}$ and $I$ have the same radical if one adopts the conditions of Corollary 5.6. However, the following simple example (taken from [Huneke 1983]) shows that [Corso et al. 2006, Corollary 2.7] is not correct, and thus Levin's result is not true.

Example 5.8. Let

$$
X=\left(\begin{array}{llll}
x_{1} & x_{3} & x_{5} & x_{7} \\
x_{2} & x_{4} & x_{6} & x_{8}
\end{array}\right)
$$

be a generic $2 \times 4$ matrix. Let $J=I_{2}(X), \mathfrak{a}=\left(x_{1} x_{4}-x_{2} x_{3}, x_{1} x_{6}-x_{2} x_{5}, x_{1} x_{8}-x_{2} x_{7}\right)$ and $I=\left(x_{1}, x_{2}\right)$. Then $J=\mathfrak{a}: I$ is a geometric 3-residual intersection of $I$ and is prime, and $\mathfrak{a}=I \cap J$, hence $\mathfrak{a}$ is radical. Furthermore, $\operatorname{Ann}\left(H_{1}(\mathfrak{a})\right)=\mathfrak{a}^{\text {unm }}=I$, which is not contained in $\mathfrak{a}$.

A simpler example would be the following degeneration of the above example. Let $\mathfrak{a}=\left(y_{1}^{2}-y_{2}^{2}, y_{1} y_{3}, y_{2} y_{4}\right) \in k\left[y_{1}, \ldots, y_{4}\right]$. Then $\operatorname{Ann}\left(H_{1}(\mathfrak{a})\right)=\left(y_{1}, y_{2}\right) \neq \operatorname{rad}(\mathfrak{a})$. 
At the end, we make a conjecture and ask a question. The conjecture will imply in turn the Cohen-Macaulayness of algebraic residual intersections for ideals with the sliding depth condition.

Conjecture 5.9. If $(R, \mathfrak{m})$ is a $C M$ local ring of dimension $d$ and I satisfies SD with depth $(R / I) \geq d-s$, then the disguised s-residual intersection of I coincides with the algebraic $s$-residual intersection.

Here is a peculiar question that arises from Corollary 5.6.

Question 5.10. Does the equality hold in $(\star)$, if $s>\mathrm{ht}(I)$ ?

Finally, we present, without proof (a proof is based on Proposition 5.1), the following proposition which is an effort toward Question 5.10.

Proposition 5.11. Let $(R, \mathfrak{m})$ be a $C M$ local ring of dimension $d$, and let $\mathfrak{a}=$ $\left(f_{1}, f_{2}, \ldots, f_{s}\right)$ and $I=\left(f_{1}, f_{2}, \ldots, f_{r}\right)$ be ideals with $s \leq r$ and $\mathrm{ht}(I)=\mathrm{ht}(\mathfrak{a}) \geq 1$. Suppose that $\mathrm{ht}(\mathfrak{a}: I) \geq n \geq 1$.

- If $s+1 \leq r$ and $\operatorname{depth}(R / I) \geq d-n+1$, then $\operatorname{Ann}\left(H_{j}\left(f_{1}, f_{2}, \ldots, f_{r}\right)\right)=I$ for $0 \leq j \leq r-s$.

- If I satisfies $\mathrm{SD}$, then, for $\mu \geq s$ and $\mu-g \geq j \geq \mu-n+2$,

$$
\operatorname{Ann}\left(H_{j}\left(f_{1}, f_{2}, \ldots, f_{\mu}\right)\right)=\bigcap_{i=0}^{\mu-s} \operatorname{Ann}\left(H_{j-i}\left(f_{1}, f_{2}, \ldots, f_{s}\right)\right) .
$$

\section{Acknowledgment}

The authors would like to thank Bernd Ulrich for encouragement and illuminating questions during his stays in Brazil in 2013 and 2014. A part of this work was done while Hassanzadeh was visiting the University of Utah, supported by the PDE-fellowship 233035/2014-1 from CNPq-Brazil. He expresses his gratitude to $\mathrm{CNPq}$ and thanks the University of Utah for the hospitality. This work is a part of Naéliton's Ph.D. thesis at IMPA, Rio de Janeiro; he would like to thank IMPA for all of its support during the preparation of this work. Thanks to E. Tavanfar, A. Boocher and S. Iyengar for reading the draft and correcting some important typos. We also thank the referee for his/her careful reading of the manuscript and useful comments.

\section{References}

[Artin and Nagata 1972] M. Artin and M. Nagata, "Residual intersections in Cohen-Macaulay rings", J. Math. Kyoto Univ. 12:2 (1972), 307-323. MR 0301006 Zbl 0263.14019

[Bruns and Herzog 1998] W. Bruns and J. Herzog, Cohen-Macaulay rings, 2nd ed., Cambridge Studies in Advanced Mathematics 39, Cambridge University Press, 1998. MR 1251956 Zbl 0909.13005 
[Chardin et al. 2001] M. Chardin, D. Eisenbud, and B. Ulrich, "Hilbert functions, residual intersections, and residually $S_{2}$ ideals”, Compositio Math. 125:2 (2001), 193-219. MR 1815393 Zbl 0983.13005

[Chardin et al. 2015] M. Chardin, D. Eisenbud, and B. Ulrich, "Hilbert series of residual intersections", Compos. Math. 151:9 (2015), 1663-1687. MR 3406441 Zbl 1329.13023

[Corso et al. 2006] A. Corso, C. L. Huneke, D. Katz, and W. V. Vasconcelos, "Integral closure of ideals and annihilators of homology", pp. 33-48 in Commutative algebra, edited by A. Corso et al., Lecture Notes in Pure and Applied Mathematics 244, Chapman \& Hall/CRC, Boca Raton, FL, 2006. MR 2184788 Zbl 1119.13006

[Eisenbud 1995] D. Eisenbud, Commutative algebra: with a view toward algebraic geometry, Graduate Texts in Mathematics 150, Springer, New York, NY, 1995. MR 1322960 Zbl 0819.13001

[Eisenbud and Ulrich 2016] D. Eisenbud and B. Ulrich, "Duality and socle generators for residual intersections", preprint, 2016. arXiv 1309.2050v3

[Eisenbud et al. 2004] D. Eisenbud, C. L. Huneke, and B. Ulrich, "Order ideals and a generalized Krull height theorem”, Math. Ann. 330:3 (2004), 417-439. MR 2099187 Zbl 1087.13009

[Fulton 1998] W. Fulton, Intersection theory, 2nd ed., Ergebnisse der Mathematik und ihrer Grenzgebiete (3) 2, Springer, Berlin, 1998. MR 1644323 Zbl 0885.14002

[Hartshorne 1977] R. Hartshorne, Algebraic geometry, Graduate Texts in Mathematics 52, Springer, New York, NY, 1977. MR 0463157 Zbl 0367.14001

[Hassanzadeh 2012] S. H. Hassanzadeh, "Cohen-Macaulay residual intersections and their Castelnuovo-Mumford regularity”, Trans. Amer. Math. Soc. 364:12 (2012), 6371-6394. MR 2958940 Zbl 1277.13008

[Herzog et al. 1983] J. Herzog, A. Simis, and W. V. Vasconcelos, "Koszul homology and blowingup rings", pp. 79-169 in Commutative algebra (Trento, 1981), edited by S. Greco and G. Valla, Lecture Notes in Pure and Applied Mathematics 84, Dekker, New York, NY, 1983. MR 686942 Zbl 0499.13002

[Huneke 1983] C. L. Huneke, "Strongly Cohen-Macaulay schemes and residual intersections", Trans. Amer. Math. Soc. 277:2 (1983), 739-763. MR 694386 Zbl 0514.13011

[Huneke and Ulrich 1988] C. L. Huneke and B. Ulrich, "Residual intersections", J. Reine Angew. Math. 390 (1988), 1-20. MR 953673 Zbl 0732.13004

[Stanley 1980] R. P. Stanley, "Weyl groups, the hard Lefschetz theorem, and the Sperner property", SIAM J. Algebraic Discrete Methods 1:2 (1980), 168-184. MR 578321 Zbl 0502.05004

[Ulrich 1992] B. Ulrich, "Remarks on residual intersections", pp. 133-138 in Free resolutions in commutative algebra and algebraic geometry (Sundance, UT, 1990), edited by D. Eisenbud and C. L. Huneke, Research Notes in Mathematics 2, Jones and Bartlett, Boston, MA, 1992. MR 1165323 Zbl 0790.13005

[Ulrich 1994] B. Ulrich, “Artin-Nagata properties and reductions of ideals", pp. 373-400 in Commutative algebra: syzygies, multiplicities, and birational algebra (South Hadley, MA, 1992), edited by W. J. Heinzer et al., Contemporary Mathematics 159, American Mathematical Society, Providence, RI, 1994. MR 1266194 Zbl 0821.13008

[Vasconcelos 1994] W. V. Vasconcelos, Arithmetic of blowup algebras, London Mathematical Society Lecture Note Series 195, Cambridge University Press, 1994. MR 1275840 Zbl 0813.13008

[Vasconcelos 2005] W. V. Vasconcelos, Integral closure: Rees algebras, multiplicities, algorithms, Springer, Berlin, 2005. MR 2153889 Zbl 1082.13006

[Weibel 1994] C. A. Weibel, An introduction to homological algebra, Cambridge Studies in Advanced Mathematics 38, Cambridge University Press, 1994. MR 1269324 Zbl 0797.18001 
Communicated by Craig Huneke

Received 2015-01-21 Revised 2016-01-13 Accepted 2016-03-22

hamid@im.ufrj.br

Instituto de Matemática, Universidade Federal do Rio de Janeiro, Av. Athos da Silveira Ramos 149, Centro de Tecnologia - Bloco C, Cidade Universitária - Ilha do Fundão, 68530 21941-909 Rio de Janeiro, Brazil

jnaeliton@yahoo.com.br Departamento de Matemática, CCEN, Campus I - sn Cidade Universitária, Universidade Federal de Paraíba, 58051-090 João Pessoa, Brazil 


\section{Algebra \& Number Theory}

msp.org/ant

\section{EDITORS}

MANAGING EDITOR

Bjorn Poonen

Massachusetts Institute of Technology

Cambridge, USA

\author{
EDITORIAL BOARD CHAIR \\ David Eisenbud \\ University of California \\ Berkeley, USA
}

BOARD OF EDITORS

Georgia Benkart

Dave Benson

Richard E. Borcherds

John H. Coates

J-L. Colliot-Thélène

Brian D. Conrad

Hélène Esnault

Hubert Flenner

Sergey Fomin

Edward Frenkel

Andrew Granville

Joseph Gubeladze

Roger Heath-Brown

Craig Huneke

Kiran S. Kedlaya

János Kollár

Yuri Manin

Philippe Michel
University of Wisconsin, Madison, USA

University of Aberdeen, Scotland

University of California, Berkeley, USA

University of Cambridge, UK

CNRS, Université Paris-Sud, France

Stanford University, USA

Freie Universität Berlin, Germany

Ruhr-Universität, Germany

University of Michigan, USA

University of California, Berkeley, USA

Université de Montréal, Canada

San Francisco State University, USA

Oxford University, UK

University of Virginia, USA

Univ. of California, San Diego, USA

Princeton University, USA

Northwestern University, USA

École Polytechnique Fédérale de Lausanne
Susan Montgomery

Shigefumi Mori

Raman Parimala

Jonathan Pila

Anand Pillay

Victor Reiner

Peter Sarnak

Joseph H. Silverman

Michael Singer

Vasudevan Srinivas

J. Toby Stafford

Ravi Vakil

Michel van den Bergh

Marie-France Vignéras

Kei-Ichi Watanabe

Efim Zelmanov

Shou-Wu Zhang
University of Southern California, USA

RIMS, Kyoto University, Japan

Emory University, USA

University of Oxford, UK

University of Notre Dame, USA

University of Minnesota, USA

Princeton University, USA

Brown University, USA

North Carolina State University, USA

Tata Inst. of Fund. Research, India

University of Michigan, USA

Stanford University, USA

Hasselt University, Belgium

Université Paris VII, France

Nihon University, Japan

University of California, San Diego, USA

Princeton University, USA

\section{PRODUCTION}

production@msp.org

Silvio Levy, Scientific Editor

See inside back cover or msp.org/ant for submission instructions.

The subscription price for 2016 is US $\$ 290 /$ year for the electronic version, and $\$ 485 /$ year ( $+\$ 55$, if shipping outside the US) for print and electronic. Subscriptions, requests for back issues and changes of subscribers address should be sent to MSP.

Algebra \& Number Theory (ISSN 1944-7833 electronic, 1937-0652 printed) at Mathematical Sciences Publishers, 798 Evans Hall \#3840, c/o University of California, Berkeley, CA 94720-3840 is published continuously online. Periodical rate postage paid at Berkeley, CA 94704, and additional mailing offices.

ANT peer review and production are managed by EditFLOW ${ }^{\circledR}$ from MSP.

\section{PUBLISHED BY}

- mathematical sciences publishers

nonprofit scientific publishing

http://msp.org/

(C) 2016 Mathematical Sciences Publishers 


\section{Algebra \& Number Theory}

Volume $10 \quad$ No. $4 \quad 2016$

Moduli of morphisms of logarithmic schemes

JONATHAN WISE

Residual intersections and the annihilator of Koszul homologies

SEYED HAMID HaSSANZADEH and Jose NAÉLITON

The Prym map of degree-7 cyclic coverings

HERBERT LANGE and ANGELA ORTEGA

SIMON MARSHALL

Hasse principle for Kummer varieties

Yonatan HaRPaZ and AlEXEI N. SkoRobogatov

Analytic continuation on Shimura varieties with $\mu$-ordinary locus

STÉPHANE BIJAKOWSKI

A note on secondary $K$-theory

GONÇALO TABUADA

Nef cones of Hilbert schemes of points on surfaces

Barbara Bolognese, Jack Huizenga, Yinbang Lin, Eric Riedl,

BENJAMIN SCHMidT, MATTHEW WoOlF and XiaOlei ZHAO

Interpolation for restricted tangent bundles of general curves 